ARTICLE

\title{
Proline rich 11 (PRR11) overexpression amplifies PI3K signaling and promotes antiestrogen resistance in breast cancer
}

Kyung-min Lee (1) 1, Angel L. Guerrero-Zotano (1) 2, Alberto Servetto', Dhivya R. Sudhan', Chang-Ching Lin', Luigi Formisano², Valerie M. Jansen², Paula González-Ericsson (1) ${ }^{3}$, Melinda E. Sanders ${ }^{3}$, Thomas P. Stricker ${ }^{3}$, Ganesh Raj ${ }^{4}$, Kevin M. Dean (10 ${ }^{5}$, Reto Fiolka (10 ${ }^{5,6}$, Lewis C. Cantley (1) ${ }^{7}$, Ariella B. Hanker (1) ${ }^{1}$ \& Carlos L. Arteaga (10) 1,3凶

The 17q23 amplicon is associated with poor outcome in $\mathrm{ER}^{+}$breast cancers, but the causal genes to endocrine resistance in this amplicon are unclear. Here, we interrogate transcriptome data from primary breast tumors and find that among genes in 17q23, PRR11 is a key gene associated with a poor response to therapeutic estrogen suppression. PRR11 promotes estrogen-independent proliferation and confers endocrine resistance in $\mathrm{ER}^{+}$breast cancers. Mechanistically, the proline-rich motif-mediated interaction of PRR11 with the p85 $\alpha$ regulatory subunit of PI3K suppresses p85 homodimerization, thus enhancing insulinstimulated binding of p110-p85 $\alpha$ heterodimers to IRS1 and activation of PI3K. PRR11-amplified breast cancer cells rely on PIK3CA and are highly sensitive to PI3K inhibitors, suggesting that PRR11 amplification confers PI3K dependence. Finally, genetic and pharmacological inhibition of PI3K suppresses PRR11-mediated, estrogen-independent growth. These data suggest ER $+/ P R R 11$-amplified breast cancers as a novel subgroup of tumors that may benefit from treatment with PI3K inhibitors and antiestrogens.

\footnotetext{
${ }^{1}$ Harold C. Simmons Comprehensive Cancer Center, University of Texas Southwestern Medical Center, Dallas, TX 75390, USA. ${ }^{2}$ Department of Medicine, Vanderbilt University Medical Center, Nashville, TN 37232, USA. ${ }^{3}$ Breast Cancer Research Program, Vanderbilt Ingram Cancer Center, Vanderbilt University Medical Center, Nashville, TN 37232, USA. ${ }^{4}$ Department of Urology, University of Texas Southwestern Medical Center, Dallas, TX 75390, USA.

${ }^{5}$ Department of Cell Biology, University of Texas Southwestern Medical Center, Dallas, TX 75390, USA. ${ }^{6}$ Lyda Hill Department of Bioinformatics, University of Texas Southwestern Medical Center, Dallas, TX 75390, USA. ${ }^{7}$ Meyer Cancer Center, Weill Cornell Medicine College, New York, NY 10065, USA.

凶email: Carlos.Arteaga@UTSouthwestern.edu
} 
pproximately $80 \%$ of breast cancers are estrogen receptor (ER)-positive and depend on estrogen for growth ${ }^{1}$. Therapies for $\mathrm{ER}^{+}$breast cancer inhibit ER signaling by directly antagonizing ER (i.e., fulvestrant) or by abolishing estrogen production (i.e., aromatase inhibitors). Adjuvant anti-ER therapies significantly reduce the risk of recurrence in patients with $\mathrm{ER}^{+}$breast cancer ${ }^{2}$. However, approximately $20 \%$ of patients treated with adjuvant endocrine therapy eventually relapse with metastatic disease ${ }^{3}$. To date, several mechanisms of de novo and acquired resistance to endocrine therapy have been reported 4 . Due to advances in large-scale tumor DNA sequencing, several somatic alterations that promote endocrine resistance have been discovered. Mutations in the ligand-binding domain of ESR1, the gene encoding ERa, confer resistance to estrogen suppression via ligand-independent ERa transcriptional activity $^{5}$. Amplification of growth factor receptors such as ERBB2 and FGFR1 has also been associated with endocrine therapy resistance ${ }^{6,7}$. Enrichment of CCND1 amplification in luminal B tumors also suggests a potential causal role with a drug-resistant phenotype $^{1}$. More recently, Razavi and colleagues reported that mutations in components of the mitogen-activated protein kinase (MAPK) pathway and the ER transcriptional program, found in approximately $20 \%$ of $\mathrm{ER}^{+}$breast cancers, are associated with shorter response to antiestrogen therapy ${ }^{8}$. Preclinical and clinical studies have suggested a critical role for hyperactivation of the phosphoinositide 3-kinase (PI3K)/AKT pathway in endocrine resistance $^{9-12}$. In line with this causal role, the PI3Ka inhibitor alpelisib in combination with the ER antagonist fulvestrant was clearly superior than fulvestrant alone in patients with advanced $\mathrm{ER}^{+} /$PIK3CA mutant breast cancer ${ }^{13}$, leading to the approval of alpelisib + fulvestrant in this subgroup of $\mathrm{ER}^{+}$breast cancers.

We recently reported genomic profiling of $\mathrm{ER}^{+}$breast tumors after short-term treatment with the aromatase inhibitor (AI), letrozole $^{14}$. In this study, the $11 \mathrm{q} 13.3,8 \mathrm{p} 11.23$, and $17 \mathrm{q} 21-23$ amplicons significantly correlated with high levels of the proliferation marker Ki67 upon drug-induced estrogen suppression. FGFR1 and CCND1 amplification, in 8p11-12 and 11q13, respectively, were associated with resistance to letrozole as defined by maintenance of a high Ki67 score on treatment. Although the 17q23 amplicon has been associated with highly proliferative luminal $\mathrm{B}$ tumors and high risk of recurrence in $\mathrm{ER}^{+}$ breast cancers ${ }^{15,16}$, a specific gene or genes in this region that would be causal to endocrine resistance have not been uncovered. In a recent study, we performed whole transcriptome analysis on RNA extracted from $58 \mathrm{ER}^{+}$breast cancers from patients treated with prolonged neoadjuvant letrozole ${ }^{17}$. In this cohort, we identified PRR11 (Proline rich 11), a protein-coding gene located in chromosome $17 \mathrm{q} 22-23$, to be overexpressed in tumors resistant to estrogen suppression compared to letrozole-sensitive tumors. PRR11 has been implicated in poor outcome of various cancer types $^{18-20}$, but the molecular basis for this association is unclear. We hypothesized that PRR11 amplification in the 17q23 amplicon promotes endocrine resistance in $\mathrm{ER}^{+}$breast cancer. We show herein that high $P R R 11$ is causally associated with estrogenindependent growth of $\mathrm{ER}^{+}$breast cancer cells. This action involved a PR (proline rich) domain-dependent interaction of PRR11 with the p85 regulatory subunit of PI3K which reduces homodimerization of $\mathrm{p} 85$ and, in turn, is permissive of ligand-induced association of $\mathrm{p} 110 \mathrm{a}$ with insulin receptor substrate 1 (IRS1) and activation of PI3K. Ectopic expression of PRR11 failed to promote estrogen-independent growth when p110a was knocked down, and PRR11-overexpressing cells were highly sensitive to PI3K inhibitors, suggesting that PRR11 amplification generates dependence on PI3K signaling, particularly in the setting of estrogen deprivation. Taken together, these data suggest a combination of PI3K and ER $\alpha$ targeted therapies is a rational approach against $\mathrm{ER}^{+}$breast cancers with PRR11 amplification.

\section{Results}

PRR11 is associated with poor outcome of $\mathrm{ER}^{+}$breast cancers. In order to identify genes associated with poor outcome of $\mathrm{ER}^{+}$ berast cancers treated with antiestrogens, we had performed whole transcriptome analysis on RNA extracted from $58 \mathrm{ER}^{+}$ breast cancers from patients treated with long-term letrozole for a median of 7.2 months (Supplementary Table 1; cohort of Guerrero-Zotano et al. ${ }^{17}$ ). PRR11 mRNA was significantly upregulated in resistant tumors. In this study, resistance to estrogen suppression was defined by a preoperative endocrine prognostic index $(\mathrm{PEPI}) \geq 4$ and/or evidence of cancer relapse after a median follow-up of 5 years [ $\log 2$ fold change $>1$ and false discovery rates $($ FDR $)<0.05$; Fig. 1a]. RNA-seq analysis of the treated tumors showed that among 51 genes in $17 \mathrm{q} 23, P R R 11$ was the only gene significantly overexpressed in resistant vs. sensitive cancers $\left(\log _{2}\right.$ fold change $=1.15, F D R=0.004, p=1.02 \mathrm{E}-05$; Fig. 1b). Patients with PRR11-high cancers displayed an increased risk of relapse in the same cohort [hazard ratio $(\mathrm{HR})=3.753$; 95\% confidence interval (CI), 1.045-13.47; Fig. 1c]. In the Kaplan-Meier Plotter database ${ }^{21}$, high PRR11 mRNA levels were also associated with a shorter relapse-free survival (RFS) of ER + /HER2 - breast cancers treated with endocrine therapy $(\mathrm{HR}=$ 3.85; 95\% CI, 1.95-7.59; Fig. 1d), but this association was not present in patients with HER2 ${ }^{+}$or triple-negative breast cancer (TNBC; Supplementary Fig. 1a). We further interrogated the association of PRR11 expression with response to estrogen suppression in two other clinical studies of $\mathrm{ER}^{+}$breast cancers treated with a neoadjuvant aromatase inhibitor (Supplementary Table 1; cohort of Giltnane et al. ${ }^{14}$; cohort of Miller et al. ${ }^{22}$ ). In these studies, maintenance of a high $\mathrm{Ki} 67$ index on treatment was used as a surrogate of resistance to estrogen suppression. In these two cohorts, we also found a statistically significant correlation between PRR11 mRNA and on-treatment high Ki67 levels (Fig. 1e, f). To correlate PRR11 expression with response to estrogen suppression, PRR11 protein levels were evaluated by IHC in tumor sections from a previously reported cohort of 175 $\mathrm{ER}^{+}$breast cancers treated with letrozole before surgery (NCT00651976 $\left.{ }^{14}\right)$. Based on the exponential curve of PRR11 protein levels, tumors were classified as PRR $11 \leq 1 \%$ (negative), $1-15 \%$ (positive) and $>15 \%$ (high; Supplementary Fig. 1b). PRR11 protein levels were statistically higher in tumors with poor response to letrozole, as defined by on-treatment high Ki67 levels ${ }^{14}$ (Fig. 1g, h).

PRR11 has been reported as an estrogen-responsive gene ${ }^{23}$. Thus, we examined the possibility that high PRR11 mRNA remained high in resistant tumors because ER is not sufficiently suppressed by letrozole treatment. In another cohort of $18 \mathrm{ER}^{+}$ breast tumors treated with an aromatase inhibitor (cohort of Miller et al. ${ }^{22}$ ), PRR11 mRNA was not downregulated in posttreatment compared to pre-treatment tumors (Supplementary Fig. 1c), implying PRR11 is not regulated by ligand-induced ER in vivo. Moreover, PRR11 mRNA levels did not correlate with estrogen response gene set signatures in three cohorts of tumors treated with an aromatase inhibitor (cohorts of Guerrero-Zotano et al., Giltnane et al., and Miller et al.; Supplementary Fig. 1d). Consistent with these correlations, exogenous estrogen did not increase PRR11 mRNA levels in MCF7 and HCC1428 cells (Supplementary Fig. 1e).

PRR11 is a key gene associated with endocrine resistance. The cytogenetic band of PRR11 is designated $17 \mathrm{q} 22$ or $17 \mathrm{q} 23.2$ (Ensembl or HGNC, respectively), located at the terminal region 
a
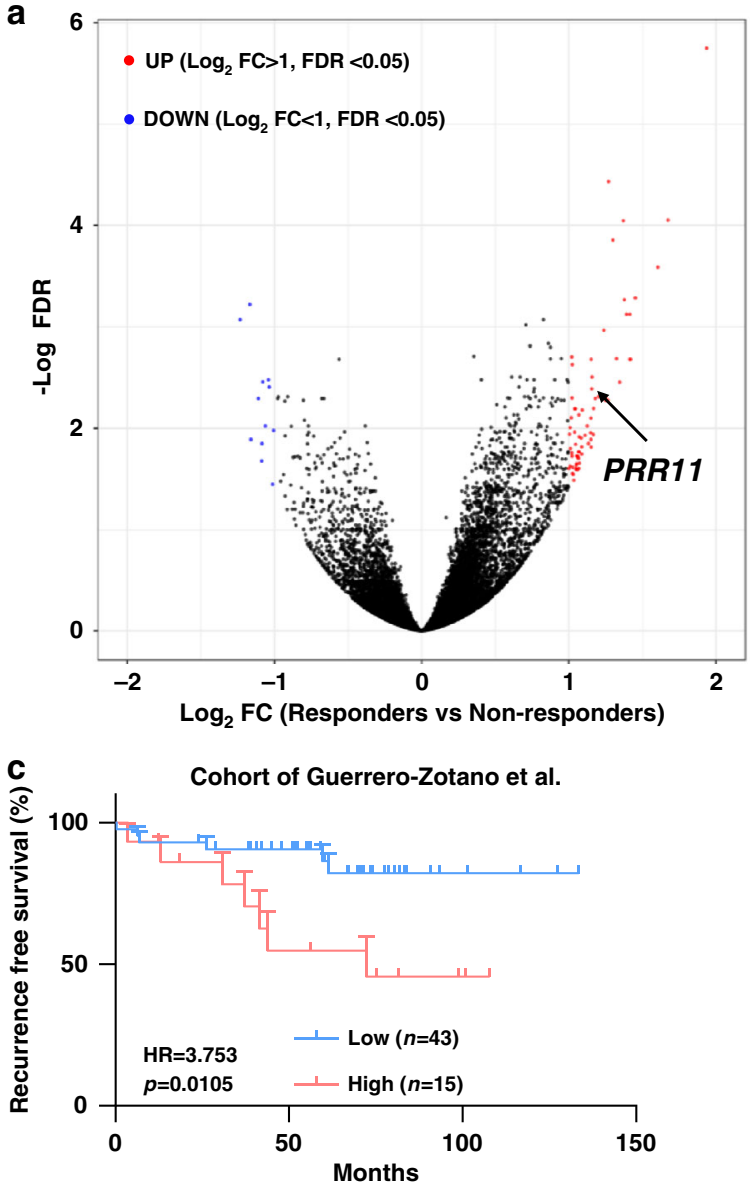

d

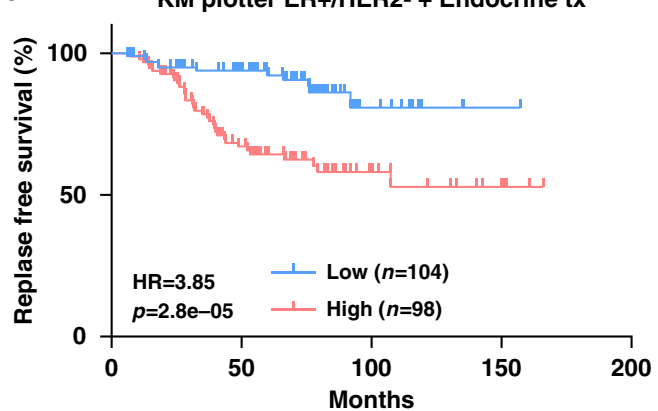

g

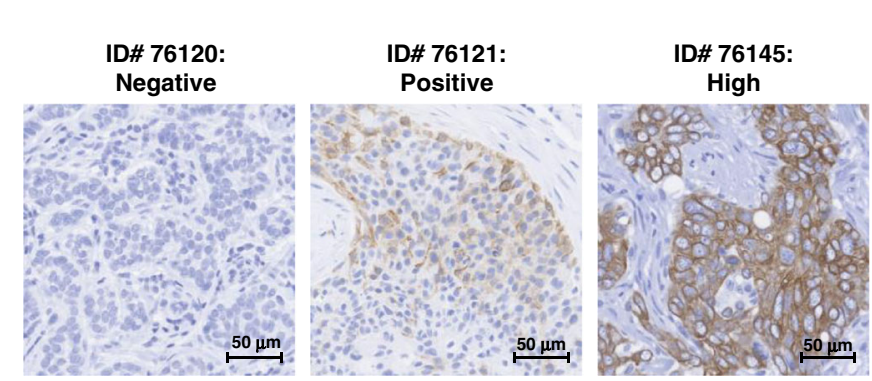

b

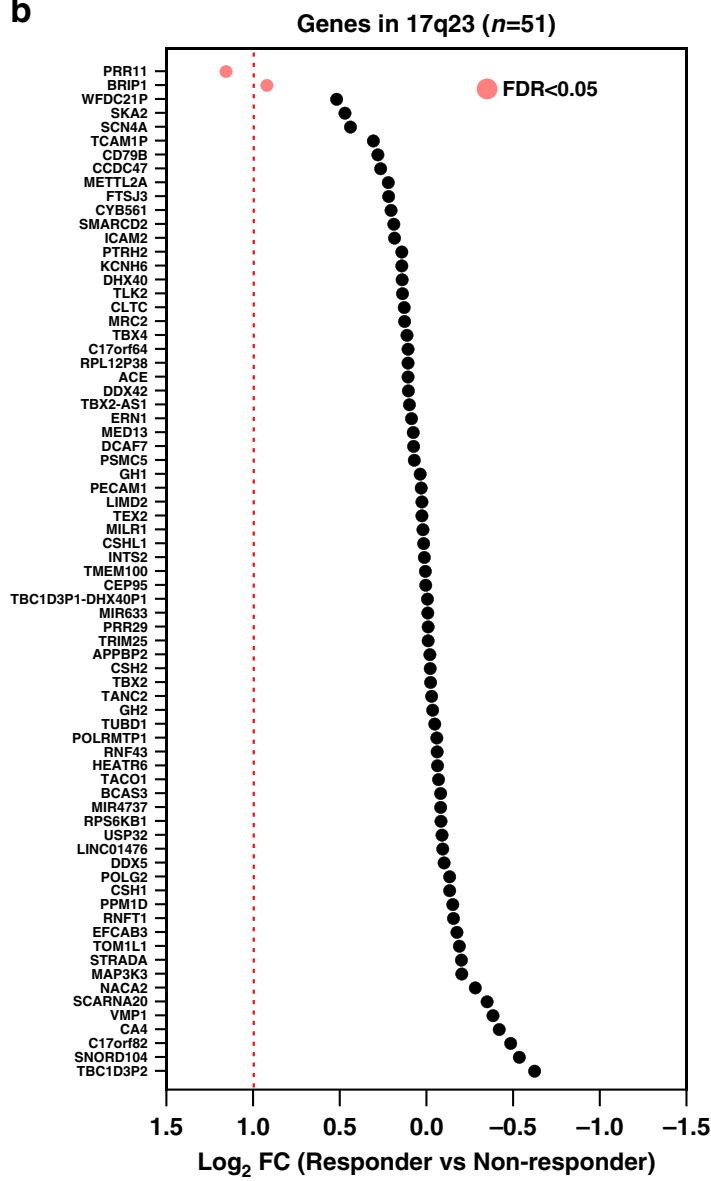

f Miller et al. $(n=18)$
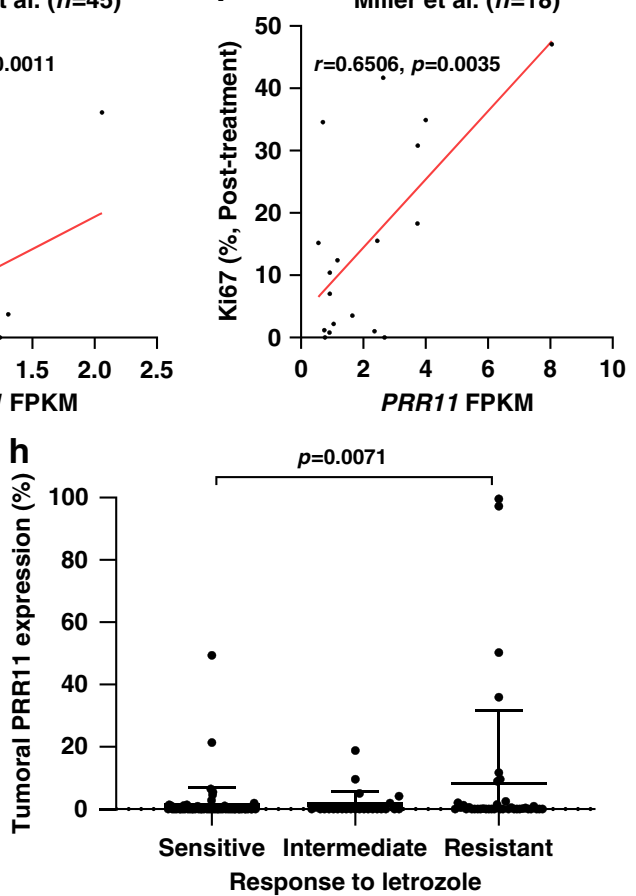

of $17 \mathrm{q} 22$ close to the $17 \mathrm{q} 23$ region (Supplementary Fig. 2a). The rate of PRR11 amplification is $15.9 \%$ in the Metastatic Breast Cancer (MBC) project, but $9.5 \%$ and $9.4 \%$ in METABRIC and The Cancer Genome Atlas (TCGA), respectively (Fig. 2a). These last two cohorts comprise primary breast tumors, thus suggesting a higher rate of PRR11 amplification in metastatic (in the MBC project) compared to $\mathrm{ER}^{+}$primary breast tumors. Of note, most metastatic $\mathrm{ER}^{+}$breast cancers have undergone adjuvant 
Fig. 1 PRR11 is associated with poor clinical outcome of ER ${ }^{+}$breast cancers treated with antiestrogens. a Volcano plot of genes differentially expressed in non-responding tumors compared to responding tumors. $\log _{2}$ fold change (FC) and false discovery rates (FDR) were calculated using DeSeq2 package. b $\log _{2} \mathrm{FC}$ of $17 \mathrm{q} 23$ locus genes in ER+/HER2- breast cancers treated with long-term letrozole $(n=51)$. Genes in 17q23 locus were selected based on the Atlas of Genetics and Cytogenetics ${ }^{25}$. Red bars indicate genes with FDR $<0.05$. c Recurrence-free survival in ER ${ }^{+}$breast cancers, treated with long-term neoadjuvant letrozole, with low or high PRR11 mRNA levels. A PRR11 FPKM cut-off (3.93) obtained from the human protein atlas was used to divide PRR11high $(n=43)$ and -low $(n=15)$ tumors (https://www.proteinatlas.org/ENSG00000068489-PRR11/pathology/breast+cancer). The Mantel-Cox model was used to calculate the hazard ratio (HR) and $p$ value. $\mathbf{d}$ Relapse-free survival of $\mathrm{ER}^{+} / \mathrm{HER} 2-$ breast cancers, treated with endocrine therapy, with low ( $n$ $=104)$ or high $(n=98)$ PRR11 mRNA levels by the auto select best cutoff in Kaplan-Meier Plotter. HR and $p$ were adopted from the Kaplan-Meier Plotter (http://kmplot.com/analysis/). e, $\mathbf{f}$ Correlation between the on-treatment percent of Ki67+ tumor cells and PRR11 mRNA level in breast tumors from the cohort of Giltnane et al. and Miller et al. (Pearson correlation). $\mathbf{g}$ Representative PRR11 immunohistochemistry (IHC) images of primary ER ${ }^{+}$breast tumors. h PRR11 levels were plotted as a function of response to estrogen suppression with letrozole in trial NCT00651976 ${ }^{14}$. Data represent the mean \pm SD $(n=$ 91, 25, 39 for drug sensitive, intermediate and resistant group, respectively; two-tailed unpaired $t$-tests). Source data are provided as a Source data file.

a

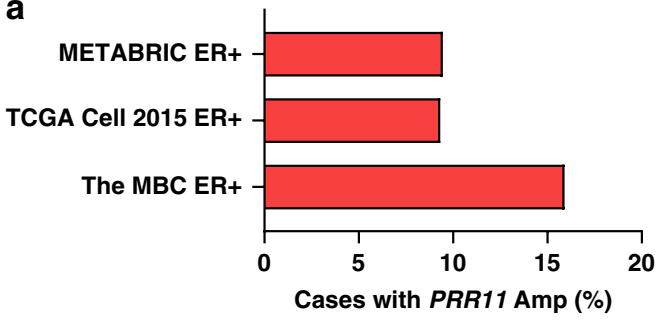

C

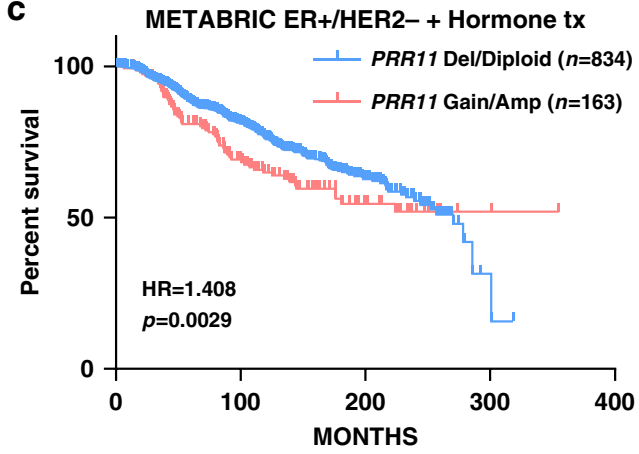

d

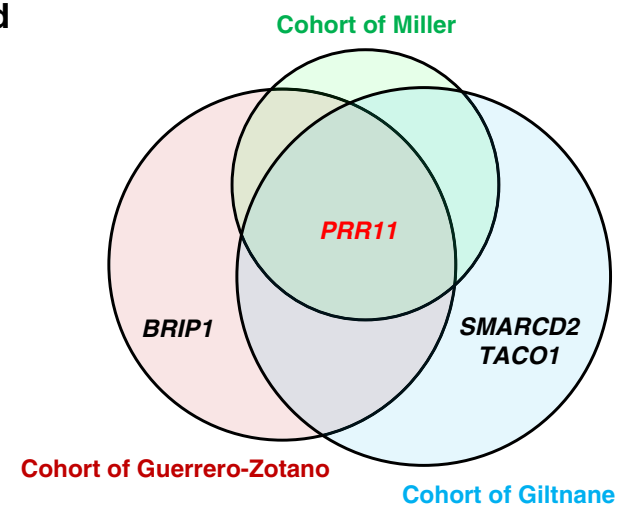

b The MBC project $(E R+/ M e t a s t a t i c / n=44)$

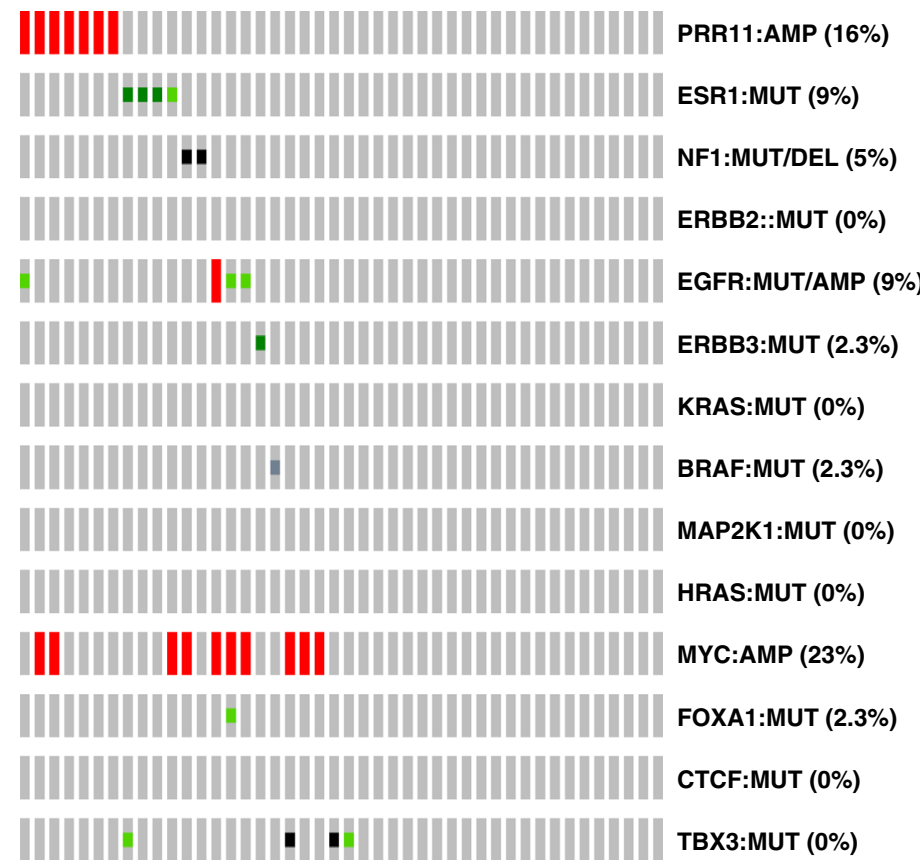

Missense (Putative driver)

Truncating (Unknown significance)

Missense (Unknown significance)

Amplification

Truncating (Putative driver)

Fig. 2 PRR11 is a key gene in 17q23 associated with endocrine resistance. a Frequency of PRR11 amplification in MBC project, TCGA or METABRIC ER ${ }^{+}$ breast cancers. b Oncoprint of putative endocrine-resistant drivers in metastatic $\mathrm{ER}^{+}$tumors from $M B C$ project dataset. ER ${ }^{+}$tumors classified as 'METASTATIC DISEASE PRESENT' were interrogated $(n=44)$. c Plot of disease-free survival of METABRIC ER ${ }^{+} / \mathrm{HER}^{-}$breast cancer patients treated with anti-hormone therapy as a function of PRR11 copy number [gain/amplification $(n=163)$ vs. diploid/deletion $(n=834)$ ]. $\mathbf{d}$ Venn diagram of genes in 17q23 that significantly correlated with on-treatment Ki67 levels (Pearson $r>0.4 ; p<0.05$ ). Source data are provided as a Source data file.

treatment with endocrine therapy, so metastatic $\mathrm{ER}^{+}$tumors often harbor somatic alterations associated with endocrine resistance. In $\mathrm{ER}^{+}$tumors in the MBC project dataset, PRR11 amplification was mutually exclusive with mutations in ESR1 and NF1, both established mechanisms of antiestrogen resistance (Fig. 2b). In the METABRIC cohort, PRR11 copy number gain or amplification predicted shorter disease-free survival of patients with $\mathrm{ER}^{+} / \mathrm{HER} 2$ - breast cancer treated with antiestrogens (HR $=1.408$; 95\% CI, 1.029-1.926; Fig. 2c). Also in METABRIC, PRR11 copy number alterations (CNAs) were correlated with PRR11 mRNA expression (Supplementary Fig. 2b). In a panel of 56 breast cancer cell lines in the CCLE dataset ${ }^{24}$, high PRR11 
copy number was significantly correlated with PRR11 mRNA (Supplementary Fig. 2c). Moreover, we observed that PRR11amplified breast cancer cell lines express higher levels of PRR11 protein compared to non-amplified cell lines $(n=11$; Supplementary Fig. 2d). We finally verified PRR11 amplification by fluorescence in situ hybridization (FISH) in a letrozole-resistant primary breast cancer (Supplementary Fig. 2e).

The gene(s) in the 17q23 amplicon that are causal to endocrine resistance have not been identified. Using the Atlas of Genetics and Cytogenetics ${ }^{25}$, we found 90 genes located in the $17 \mathrm{q} 23$ amplicon (Supplementary Table 2). We next examined the association of these genes with on-treatment Ki67 levels in three clinical studies of $\mathrm{ER}^{+}$breast cancers treated with a neoadjuvant aromatase inhibitor (Supplementary Table 1; cohort of GuerreroZotano $^{17}$; cohort of Giltnane ${ }^{14}$; cohort of Miller $^{22}$ ). This analysis revealed that four of these 90 genes in 17q23 (PRR11, BRIP1, SMARCD2, and TACO1) correlated statistically with ontreatment Ki67 levels (Pearson $r>0.4, p<0.05$; Fig. 2d; Supplementary Tables 3-5). Across these three studies, PRR11 was the only one of these four genes that exhibited a significant correlation with a high Ki67 score. Finally, among 67 genes in 17q23, high PRR11 mRNA levels also correlated with a shorter RFS in patients with $\mathrm{ER}^{+} / \mathrm{HER} 2^{-}$breast cancer treated with endocrine therapy in the Kaplan-Meier Plotter database (Supplementary Fig. 2f). Collectively, these data suggest that PRR11 may be a key gene in 17q23 associated with endocrine resistance.

Several genes in the $17 \mathrm{q} 23$ amplicon have been speculated to be associated with poor outcome in breast cancer ${ }^{15,26}$. To determine whether PRR11 is an essential gene in 17q23-amplified breast cancer cells, we interrogated genome-scale RNAi screening data of MCF7 cells that harbor $17 \mathrm{q} 23$ amplification ${ }^{27}$ in Project Achilles dataset (v2.4.3) 28 . shRNAs targeting 47 genes in $17 \mathrm{q} 23$ were screened in Project Achilles dataset. Of these 47 genes, PRR11 displayed the lowest Analytic Technique for Assessment of RNAi by Similarity (ATARiS) ${ }^{29}$ score, thus implying a high dependency of 17q23-amplified breast cancer cells on PRR11 (Supplementary Fig. 2g).

PRR11 overexpression confers resistance to antiestrogens. To further prioritize genes in $17 \mathrm{q} 23$ associated with resistance to estrogen suppression, we transduced PRR11, BRIP1, SMARCD2, and TACO1 into MDA-MB-134VI and MDA-MB-175VII cells, which do not harbor 17q23 amplification (Fig. 3a). PRR11 was the only gene that promoted growth of both cell lines under conditions of estrogen deprivation (Fig. 3b). Next, we employed MCF7 LTED (long-term estrogen deprived) and HCC1428 LTED cells 9 . MCF7 and HCC1428 wild type cells harbor PRR11 high copy number ${ }^{24}$. PRR11 ablation by siRNA abolished estrogenindependent growth of MCF7 LTED and HCC1428 LTED cells (Fig. 3c). The inhibitory effect of PRR11 ablation was rescued by re-expression of PRR11 (Fig. 3d, e). To assess the role of PRR11 in vivo, we generated xenografts of MCF7 cells stably expressing a doxycycline-inducible PRR11 shRNA in ovariectomized athymic mice. Treatment with doxycycline markedly reduced PRR11 protein levels and arrested growth of established MCF7 xenografts expressing PRR11 shRNA but not a control shRNA (Fig. $3 \mathrm{f}$ and Supplementary Fig. 3a).

In the PRISM repurposing 19Q3 dataset ${ }^{30}$, PRR11-amplified $\mathrm{ER}^{+}$breast cancer cell lines displayed a lower sensitivity to the ER antagonist fulvestrant compared to cells without PRR11 amplification (Fig. 3g). Transduction of PRR11 into MDA-MB-134VI and MDA-MB175VII cells attenuated growth inhibition by fulvestrant (Fig. 3h and Supplementary Fig. 3b). PRR11 knockdown re-sensitized fulvestrant-resistant (FulvR) MCF7 cells to fulvestrant (Fig. 3i and Supplementary Fig. 3c). A similar result was observed in tamoxifen-resistant (TamR) MCF7 and TamR HCC1428 cells (Supplementary Fig. 3d). These data suggest PRR11 promotes resistance to antiestrogens and its downregulation enhances the action of ER-targeted therapies against $\mathrm{ER}^{+}$breast cancer cells.

PRR11 promotes proliferation of $\mathrm{ER}^{+}$breast cancer cells. We next analyzed RNA-seq data in the cohort of Guerrero-Zotano et al. ${ }^{17}$ using 125 previously reported breast cancer-related gene expression signatures ${ }^{31}$. Nine proliferation-associated signatures were significantly enriched in tumors with high PRR11 mRNA expression (FDR $<0.01$; Fig. 4a). Hallmark gene sets associated with proliferation, including "E2F_TARGETS" and "G2M_CHECKPOINT" were significantly enriched in ER +/HER2 ${ }^{-}$tumors with PRR11 gain or amplification in METABRIC and in $\mathrm{ER}^{+}$tumors with PRR11 amplification in TCGA (Supplementary Fig. 4a, b).

To inquire further into the mode of action of PRR11 in cell proliferation, we determined the expression of 84 cell cycleassociated genes using a PCR array. Six genes were reduced upon transfection of PRR11 siRNA into MCF7 LTED cells (SKP2, CDKN1A, CCNB2, CCNA2, CKS2, and CCNB1; FC < 0.5; Fig. 4b). With the exception of SKP2 and CDKN1A, expression of each of these genes was significantly elevated in $\mathrm{ER}^{+}$breast cancers with PRR11 copy number gain/amplification in the METABRIC and TCGA dataset (Supplementary Fig. 4c, d). PRR11 ablation arrested cell cycle progression and inhibited proliferation in MCF7 LTED, MCF7 TamR, MCF7 FulvR, and HCC1428 LTED cells (Fig. 4c and Supplementary Fig. 4e) and these were rescued by re-expression of PRR11 (Fig. 4d). Conversely, PRR11 overexpression resulted in a marked increase in cells in $S$ phase in estrogen-deprived MDA-MB-134VI and MDA-MB-175VIII cells (Fig. 4e). Consistent with a cytostatic effect, PRR11 knockdown reduced $R B$ phosphorylation levels (Fig. 4f). Even in absence of estrogen, ectopic expression of PRR11 was capable to induce RB phosphorylation (Fig. 4g). Finally, ER transcriptional activity measured with an estrogen response element (ERE)-luciferase reporter was not affected by siRNA-mediated PRR11 ablation in parental and LTED MCF7 and HCC1428 cells (Supplementary Fig. 4f), suggesting that PRR11 confers resistance to estrogen suppression via growth-promoting signaling pathways independent of ER. PRR11 ablation did not suppress proliferation in PRR11-overexpressing triple negative and HER2 ${ }^{+}$breast cancer cells (Supplementary Fig. 4g, h).

PRR11 reduces p85 homodimers and enhances PI3K activation. Gene set signature analysis shown in Fig. 4a also revealed that PIK3CA and IGF1 signaling pathways, both reported to be associated with endocrine resistance ${ }^{9,32}$, were significantly enriched in PRR11-high tumors. In line with this association, PRR11 knockdown resulted in a reduction in phosphorylated AKT (pAKT) and p110a protein in LTED, TamR, and FulvR cells (Fig. 5a). AKT inactivates GSK3 $\beta$ through the phosphorylation, which in turn, promotes the stabilization of cyclin D1 protein $^{33,34}$. Indeed, $P R R 11$ knockdown resulted in an inactivation of GSK3 $\beta$ (Fig. 5a). PIK3CA mRNA levels were not affected by PRR11 ablation, suggesting that PIK3CA transcription is not regulated by PRR11 (Supplementary Fig. 5a). A similar reduction in p-AKT by PRR11 ablation were observed in MCF7 cells whose PIK3CA E545K allele had been corrected to a wild type sequence by somatic cell gene targeting, implying that the effect of PRR11 in PI3K activity is not limited to cells with PIK3CA mutations (Supplementary Fig. 5b-d). Conversely, overexpression of PRR11 resulted in an increase of p-AKT and p-GSK3 $\beta$ levels in MDAMB-175VII and MDA-MB-134VI cells (Fig. 5b). To determine 
a

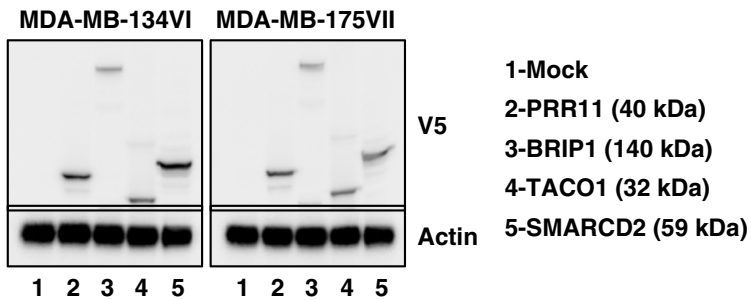

C

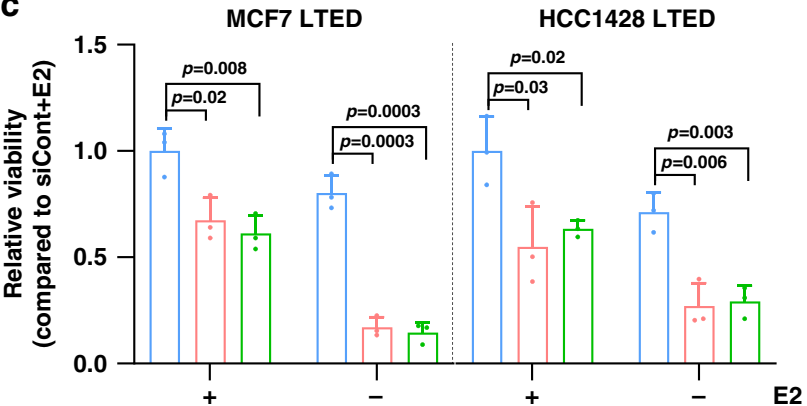

b

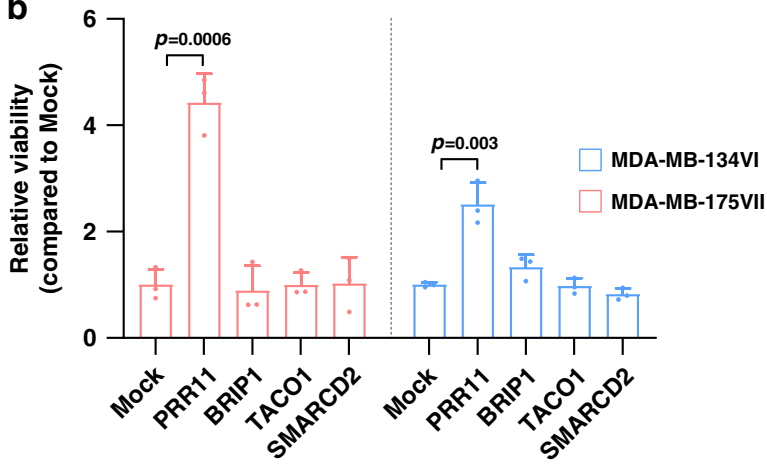

siCont

SIPRR11\#1

siPRR11\#2

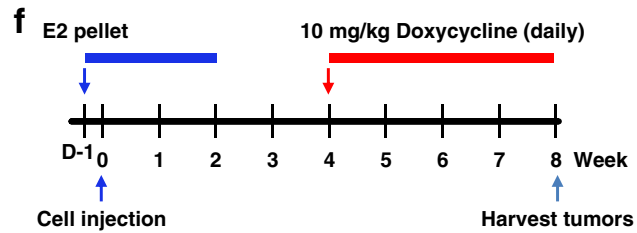

d
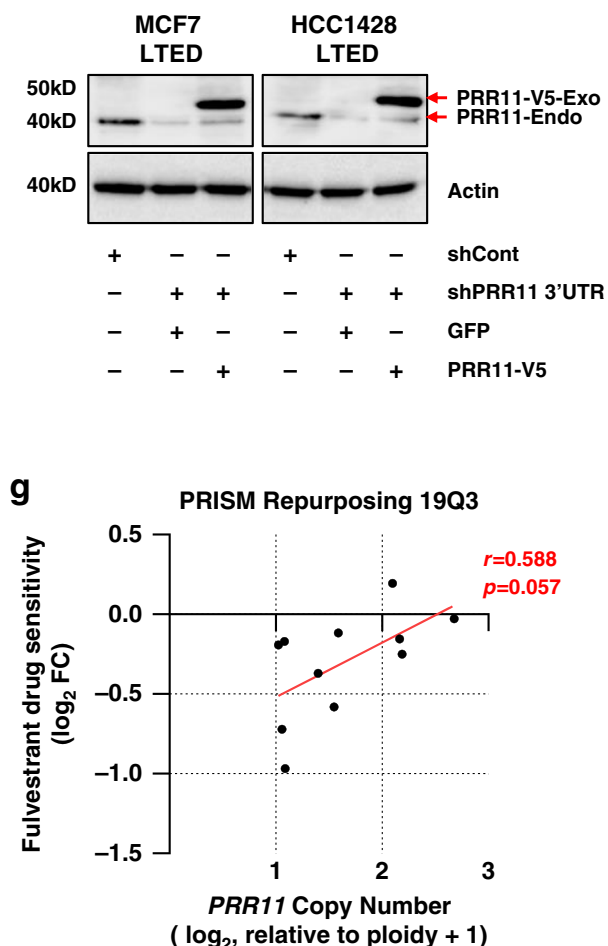

e

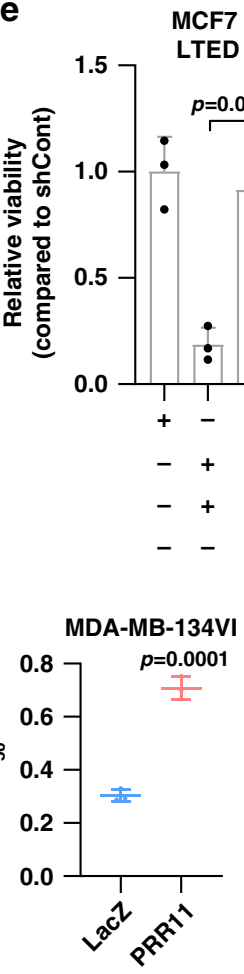

HCC1428

LTED

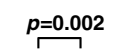

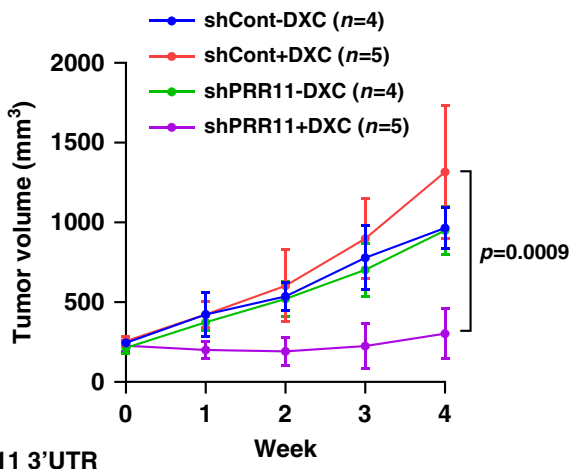

$$
\begin{aligned}
& +--+- \text { GFP } \\
& -+--+ \text { PRR11 }
\end{aligned}
$$
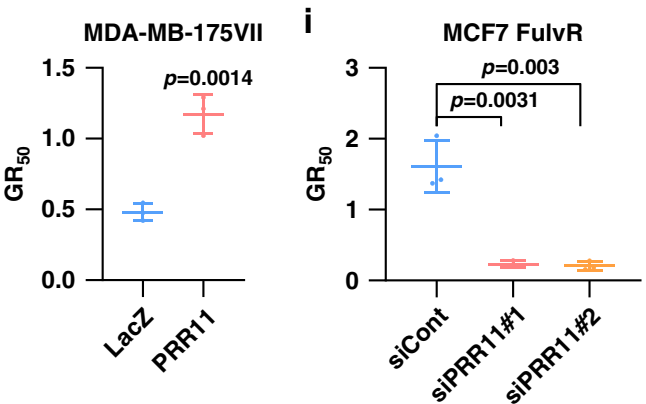

Fig. 3 PRR11 overexpression confers resistance to antiestrogens. a Lysates of MDA-MB-134VI and MDA-MB-175VII cells that had been transduced with pLX304-PRR11, -BRIP1, -TACO1, and -SMARCD2 were subjected to immunoblot analysis. b Low density monolayers of MDA-MB-134VI and MDA-MB-175VII pLX304-PRR11, -BRIP1, -TACO1, and -SMARCD2 cells were grown in estrogen-deprived condition. After 2 weeks, cell monolayers were stained with crystal violet and cell viability quantified as described in Methods. Data represent the mean \pm SD of three replicates (two-tailed unpaired $t$-tests). c MCF7 LTED and HCC1428 LTED cells were transfected with PRR11 siRNAs. Low density monolayers of cells were treated $\pm 1 \mathrm{nM}$ estrogen (E2) for 10 days. Cell monolayers were stained with crystal violet. Data represent the mean \pm SD of three replicates (two-tailed unpaired $t$-tests). d, e MCF7 LTED and HCC1428 LTED cells were transduced with shRNA targeting the 3' UTR of PRR11 and then, re-transduced with pLX304-GFP or pLX304-PRR11. Cell lysates were subjected to immunoblot analysis (d). Upper and lower arrows indicate exogenous and endogenous PRR11, respectively. Low density monolayers of cells shown in d were grown in absence of E2 for 10 days (e). Data represent the mean \pm SD of three replicates (two-tailed unpaired $t$-tests). $f$ MCF7 cells stably expressing doxycycline-inducible-PRR11 shRNA and control shRNA were injected s.c. in the dorsum of athymic ovariectomized mice supplemented with a 14-day release 17 $\beta$-estradiol pellet. After 4 weeks, mice were randomized to treatment with $10 \mathrm{mg} / \mathrm{kg}$ doxycycline (i.p) for 4 weeks. Each data point represents the mean tumor volume in $\mathrm{mm}^{3} \pm \mathrm{SD} ; n$ of mice per group are shown in parenthesis (two-tailed unpaired $t$-tests). $\mathbf{g}$ Fulvestrant sensitivity of ER ${ }^{+}$breast cancer cell lines ( $n=11$, PRISM Repurposing 19Q3 dataset). Y-axis, drug sensitivity, represents relative barcode abundance following fulvestrant treatment (Pearson correlation). $\mathbf{h}$, $\mathbf{i}$ Fulvestrant $\mathrm{GR}_{50}$ were calculated using the GR metrics calculator ${ }^{56}$ (http://www.grcalculator.org/grcalculator/). Cell numbers on days 0 and 6 were used as the input data. MDA-MB-134VI and MDA-MB-175VII cells were stably transduced with pLX302-LacZ (control) or pLX302-PRR11 (h). MCF7 FulvR cells were transfected with control or PRR11 siRNA (i). Data represent the mean \pm SD of three replicates (two-tailed unpaired $t$-tests). Source data are provided as a Source data file. 
a

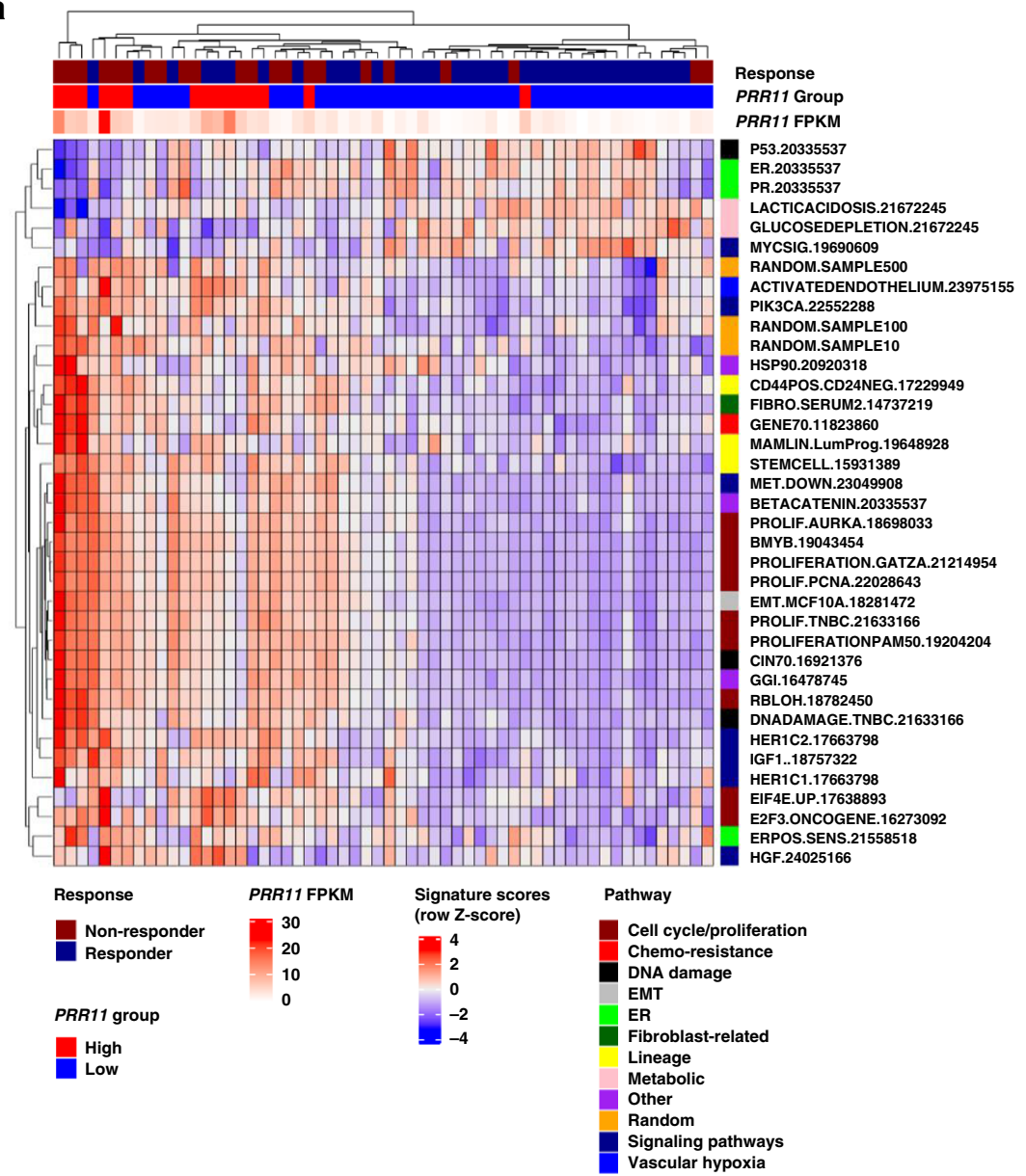

b

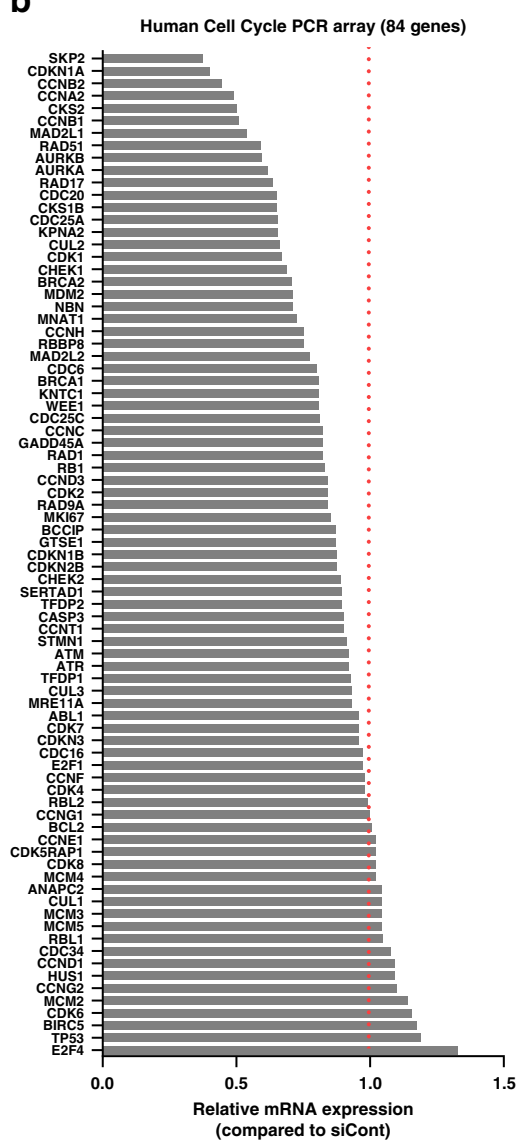

C

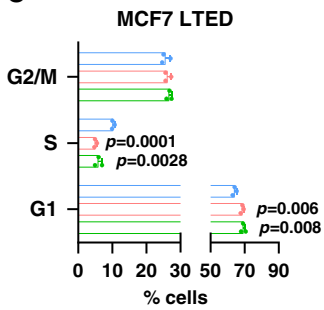

HCC1428 LTED

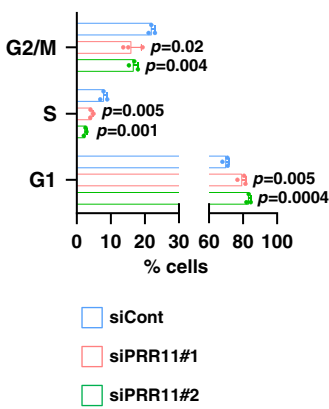

d
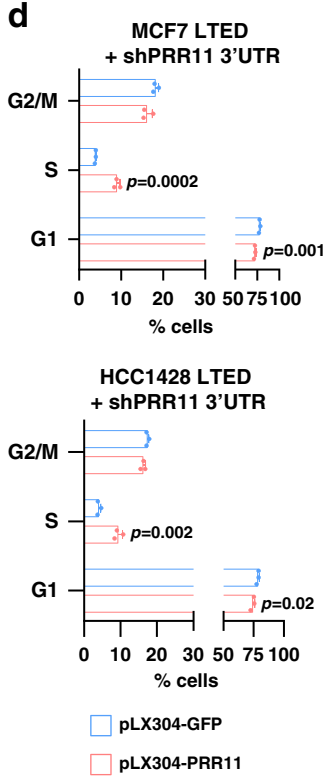

e

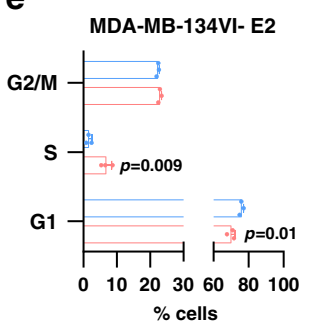

MDA-MB-175VII- E2

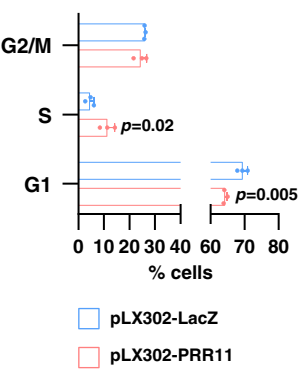

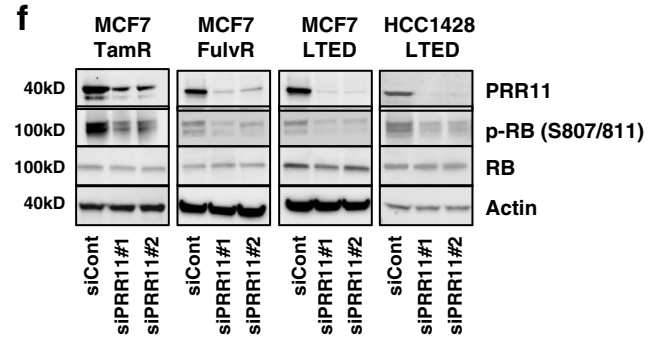

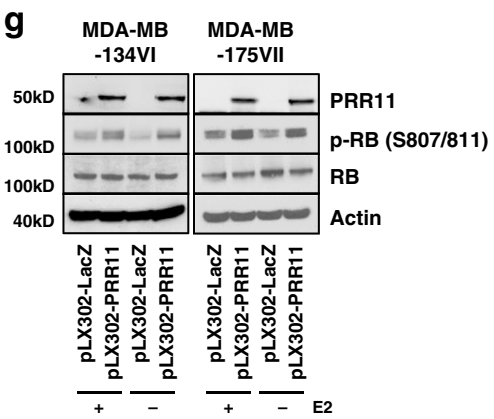

whether PRR11 affects phosphatidylinositol-3,4,5-trisphosphate (PIP3) formation, we utilized live cell imaging with a GFP-based biosensor fused with the PH domain of AKT. Detection of this biosensor at the plasma membrane is a surrogate of PI3K-induced PIP3 formation. Indeed, PRR11 silencing by siRNA resulted in a decrease of GFP-biosensor signals at the plasma membrane and membrane ruffling in MCF7 LTED cells (Supplementary Movies 1 and 2; control siRNA and siRNA targeting PRR11, respectively).

Proline-rich (PR) motifs bind to src homology 3 (SH3) domains, which are critical for the assembly of signaling complexes involved in aberrant cell proliferation ${ }^{35}$. Of potential 
Fig. 4 PRR11 overexpression enhances cancer cell proliferation. a Single sample gene set analysis was performed using a set of 125 previously reported breast cancer-related signatures. Gene sets that were differentially enriched between PRR11 high vs. low tumors (FDR < 0.01). b Complementary DNA (CDNA) of MCF7 LTED cells transfected with control or PRR11 siRNA was tested in a 84-cell cycle gene PCR array. Expression of 6 genes in the array was reduced by PRR11 siRNA transfection (FC < 0.5). Each data point represents the average of duplicate experiments. c MCF7 LTED and HCC1428 LTED cells transfected with control or PRR11 siRNA for $48 \mathrm{~h}$ were stained with propidium iodide and analyzed by flow cytometry. Data represent the mean \pm SD of three replicates (two-tailed unpaired t-tests). d MCF7 LTED and HCC1428 LTED cells transduced with shRNA targeting the 3' UTR of PRR11 and either pLX304-GFP or pLX304-PRR11 were stained with propidium iodide and analyzed by flow cytometry. Data represent the mean \pm SD of three replicates (twotailed unpaired $t$-tests). e MDA-MB-134VI and MDA-MB-175VII cells transduced with pLX302-LacZ or pLX302-PRR11 were grown in estrogen (E2)deprived condition for 4 days. Cells were stained with propidium iodide and analyzed by flow cytometry. Data represent the mean \pm SD of three replicates (two-tailed unpaired t-tests). f HCC1428 LTED, MCF7 LTED, MCF7 TamR, and MCF7 FulvR cells were transfected with control or PRR11 siRNA for $48 \mathrm{~h}$. Cell lysates were subjected to immunoblot analysis. g MDA-MB-134VI and MDA-MB-175VII cells transduced with pLX302-LacZ or pLX302-PRR11 were grown $\pm 1 \mathrm{nM}$ E2 for 4 days. Cell lysates were subjected to immunoblot analysis. Source data are provided as a Source data file.

relevance to PRR11, the p85 regulatory subunit of PI3K contains a SH3 domain and stabilizes the p110a catalytic subunit of PI3K ${ }^{36}$. Thus, to explore if PRR11 would interact with the PI3K pathway, we examined whether PRR11 associates with $\mathrm{p} 85$ via its PR motif. In MCF7 and HCC1428 cells, PRR11 was physically associated with p85a, as measured by both coimmunoprecipitation followed by immunoblot analysis and proximity ligation assay (PLA; Fig. 5c, d). This association between PRR11 and p85a was confirmed in HEK293 cells transfected with exogenous PRR11 and Flag-tagged p85a (Fig. 5d and Supplementary Fig. 6a).

The intermolecular interaction between the $\mathrm{SH} 3$ and $\mathrm{PR}$ domains in p85 monomers mediates p85 homodimerization ${ }^{37,38}$. The p85 homodimer contains four SH2 domains and, as a consequence, outcompetes p85/p110 heterodimers for binding to phosphorylated Tyr residues in insulin receptor substrate 1 (IRS1), thus inhibiting insulin/IGF-stimulated PI3K activity ${ }^{39}$. Therefore, we hypothesized that the association of surplus PRR11 with p85a would impair the formation of p85 homodimers (Fig. 5e). This would potentially enhance both the association of heterodimeric p85/p110 with IRS1 at the plasma membrane and PI3K activation stimulated by insulin/IGFs. To test this hypothesis, we co-transduced PIK3R1 (p85a) tagged with either Flag or human influenza hemagglutinin (HA) into HEK293 cells. Ectopic expression of PRR11 impaired the association between p85a-Flag and $\mathrm{p} 85 \mathrm{a}-\mathrm{HA}$ as measured by HA and Flag precipitation followed by Flag and HA immunoblot, respectively (Fig. 5f), and by PLA (Supplementary Fig. 6b), suggesting PRR11 inhibits p85 homodimerization. In MCF7 LTED cells stably expressing both PIK3R1-Flag and doxycycline-inducible PIK3R1-HA, knockdown of $P R R 11$ resulted in an increase of the p85 homodimers (Fig. $5 \mathrm{~g}$ and Supplementary Fig. 6c). PRR11 silencing also reduced insulin-mediated association of p110 $\alpha$ with IRS1 and insulin/ IGF-stimulated p-AKT in MCF7 LTED and HCC1428 LTED cells (Fig. 5h, j and Supplementary Fig. 6d). Conversely, PRR11 overexpression enhanced the p110 $\alpha$-IRS1 association and p-AKT in MDA-MB-175VII cells (Fig. 5i, j).

To determine whether the PR domain in PRR11 is crucial to the interaction of PRR11 with p85, we generated PRR11 mutant (PRR11- $\mathrm{PR}$ ) lacking the PR motif as revealed by Motif Scan, an in silico motif prediction analysis (Supplementary Fig. 7a, b). We co-transfected Flag-tagged PIK3R1 with either V5-PRR11-WT or V5-PRR11- $\triangle \mathrm{PR}$ into HEK293 cells. Immunoprecipitation with a Flag antibody revealed that deletion of the PR motif in PRR11 reduced its interaction with p85a compared to PRR11 WT (Fig. 5k), suggesting that the PR motif of PRR11 mediates the association with $\mathrm{p} 85 \mathrm{a}$. Moreover, PRR11- $\Delta \mathrm{PR}$ did not induce $\mathrm{p}$ AKT levels to the same degree as PRR11 wild type (WT) in MCF7 LTED and HEK293 cells stably transduced with PRR11 3' UTR shRNA (Supplementary Fig. 7c). Together, these data suggest that the PR motif of PRR11 promotes association with p85a, leading to reduced p85 homodimerization and enhanced PI3K activation.

PRR11 amplification is associated with PI3K activation. We next computed signature scores of gene sets associated with the insulin/PI3K pathway in $\mathrm{ER}^{+} / \mathrm{HER} 2^{-}$breast tumors in METABRIC. Signature scores of these gene sets were significantly higher in tumors harboring PRR11 gain or amplification compared to those with PRR11 deletion or diploid tumors (Fig. 6a, b). Similarly, hallmark gene sets associated with the PI3K/AKT pathway, such as "PI3K_AKT_MTOR_SIGNALING" and "MTORC1_SIGNALING", were enriched in METABRIC ER +/HER2 - breast tumors with PRR11 gain or amplification compared to PRR11 deleted/diploid tumors (Fig. 6c). We then computed the connectivity map (CMap) scores ${ }^{40}$ with the list of genes significantly upregulated in $\mathrm{ER}^{+} / \mathrm{HER} 2^{-}$breast cancers with PRR11 gain or amplification vs. diploid/deletion in METABRIC, and in $\mathrm{ER}^{+}$breast cancers with PRR11 amplification vs. no amplification in TCGA (Top 150 genes, FDR < 0.05). PI3K inhibitors and AKT signaling loss-of-function (LOF) were found as perturbation classes with $<-95$ connectivity score (tau) in MCF7 cells (Fig. 6d), representing an opposite connectivity between perturbation and gene set. This suggests that genes overexpressed in PRR11 amplified $\mathrm{ER}^{+}$breast cancers can be downregulated by perturbations that inhibit PI3K/AKT. Of note, CDK4/6 inhibitors exhibited similar opposite connectivity, suggesting these agents may also be effective against PRR11-amplified cancers.

Finally, in the METABRIC and TCGA datasets, PRR11 amplification and PIK3CA mutations were mutually exclusive of each other in $\mathrm{ER}^{+}$breast cancers. This mutual exclusivity also supports the notion that PRR11 amplification is functionally linked to aberrant activation of PI3K (Fig. 6e).

PRR11-amplified breast cancer cells rely on the PI3K pathway. The data shown so far suggest that a potential oncogenic role of PRR11 depends on activation of PI3K and, as such, PI3K inhibitors would be effective against PRR11-overexpressing breast cancer cells. To explore this, we first utilized MCF10A cells that require EGF and insulin to propagate. PIK3CA knockdown significantly inhibited the proliferation of MCF10A cells grown in media containing EGF/insulin or insulin alone (Supplementary Fig. 8a, b). PIK3CA knockdown abolished growth promoted by PRR11 overexpression, suggesting that PRR11-mediated cell growth requires PIK3CA. Consistent with these data, the PIK3CA dependence score of 57 breast cancer cell lines significantly correlated with PRR11 copy number in DEMETER2, a combined large-scale RNAi screening dataset ${ }^{41}$ (Fig. 7a). In the PRISM repurposing 19Q3 dataset ${ }^{30}$, PRR11 copy number of 27 breast cancer cell lines significantly correlated with sensitivity to the 
a

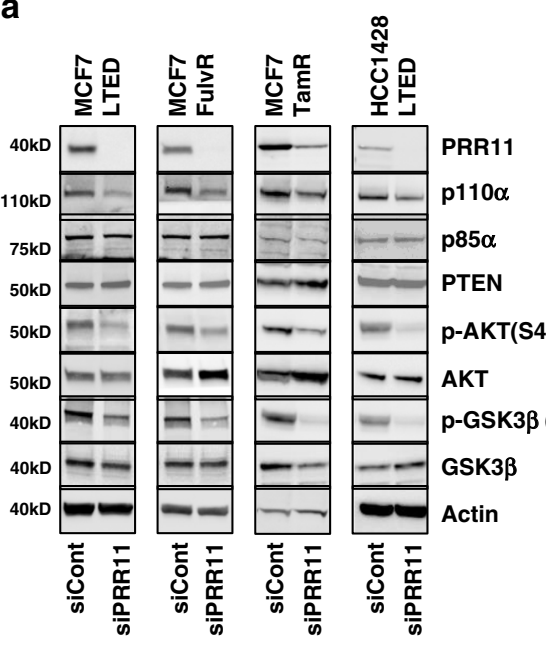

d

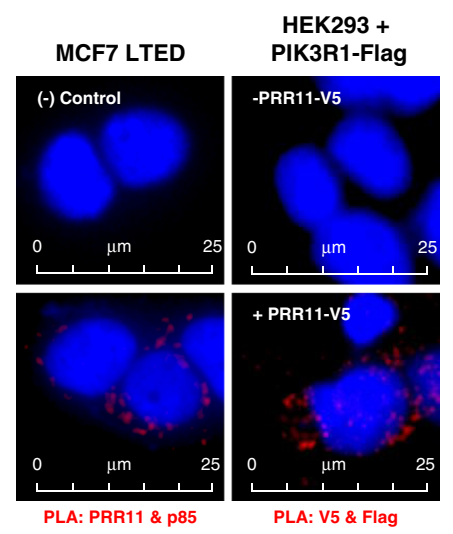

h

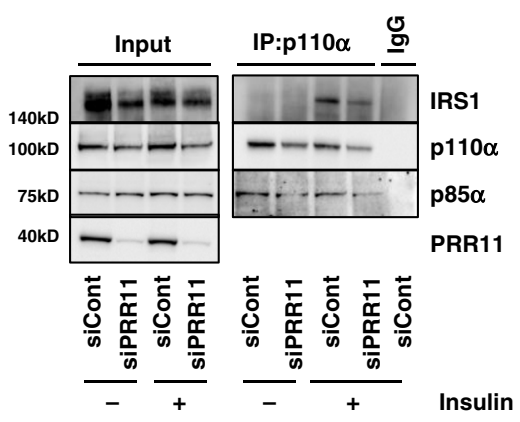

b

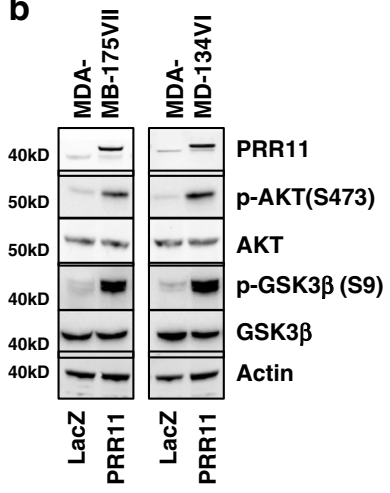

e

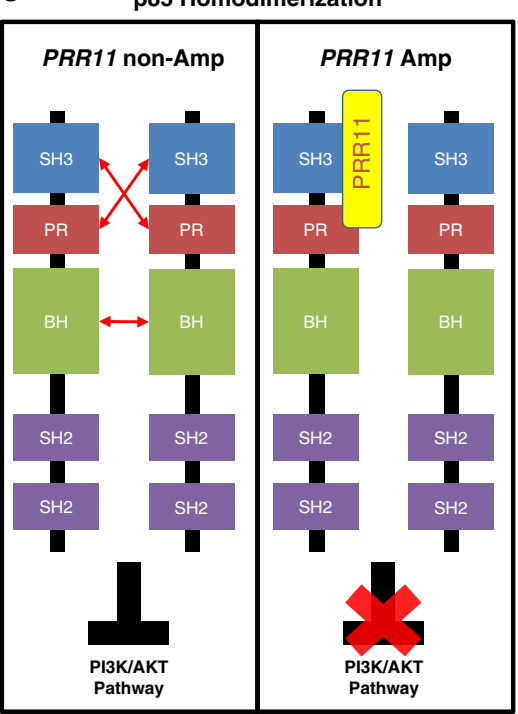

C

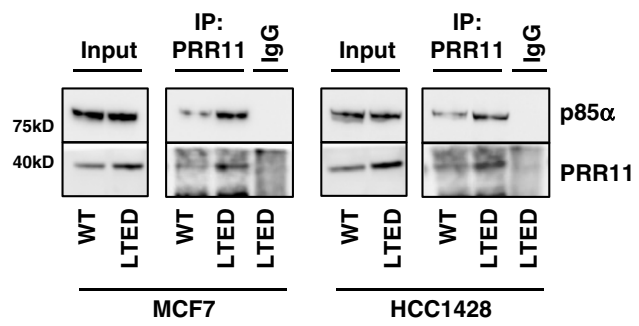

$\mathbf{f}$

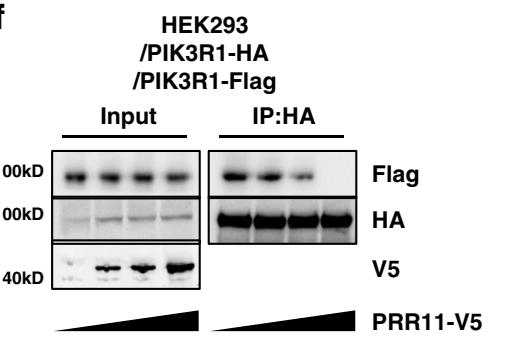

9 MCF7 LTED

/PIND-PIK3R1-HA

/pLenti6.3-PIK3R1-Flag

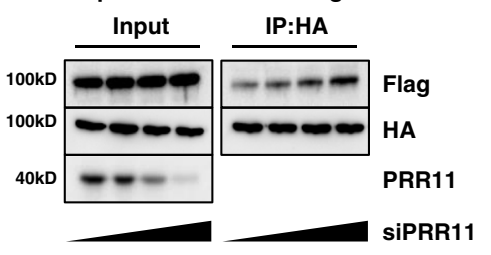

i

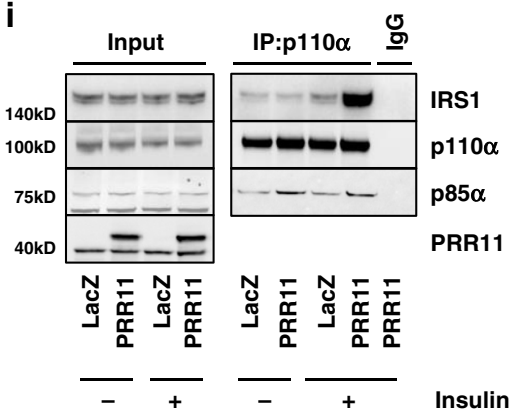

$\mathbf{k}$

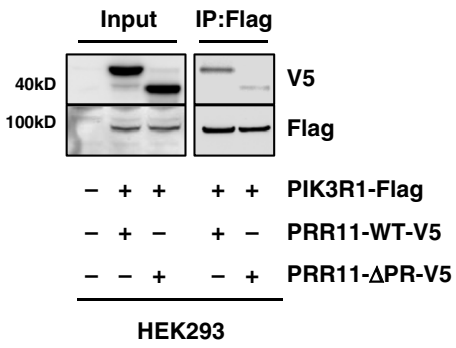

j
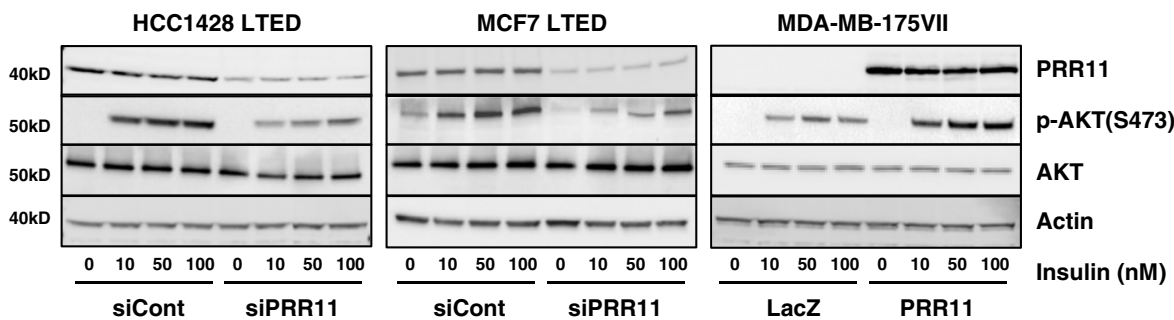

PI3K inhibitors pictilisib and taselisib (Fig. 7b). Furthermore, breast cancer cell lines with PRR11 amplification displayed significantly higher sensitivity to the PI3K inhibitor pictilisib compared to cell lines without PRR11 amplification in the LINCS MGH/Sanger dataset of Drug/Cell-line Browser (DCB ${ }^{42}$; Fig. $7 \mathrm{c}$ ). It was previously shown that ectopic expression of mutant PIK3CA sensitizes these cells to PI3K inhibitors ${ }^{43}$. Likewise,
PRR11 overexpression sensitized MCF10A and MDA-MB-134VI cells to PI3K inhibitors (Fig. 7d, e and Supplementary Fig. 8c, d). Together, these suggest that $P R R 11$-overexpressing cells rely on $P I K 3 C A$ and, as a result, are highly sensitive to PI3K inhibitors.

We finally tested whether genetic or pharmacological inhibition of PI3K would overcome PRR11-mediated resistance to estrogen suppression. PIK3CA knockdown with siRNA 
Fig. 5 PRR11 overexpression reduces p85 homodimers and enhances ligand-induced PI3K activation. a Lysates of MCF7 LTED, FulvR, TamR, and HCC1428 LTED cells transfected with PRR11 or control siRNA for $48 \mathrm{~h}$ were subjected to immunoblot analysis. b Lysates of MDA-MB-175VII and MDAMB-134VI cells transduced with pLX302-LacZ or pLX302-PRR11 were subjected to immunoblot analysis. c MCF7 parental, MCF7 LTED, HCC1428 parental, and HCC1428 LTED cell lysates were immunoprecipitated with PRR11 or IgG antibodies. Immune complexes were then subjected to immunoblot analysis. $\mathbf{d}$ MCF7 LTED cells and HEK293 cells transduced with pLenti7.3-PIK3R1-Flag and pLX302-PRR11-V5 were subjected to proximity ligation assay (PLA) with PRR11, p85 $\alpha$, V 5 and Flag antibodies. e Schema of p 85 monomers associating via a SH3-PR domain intermolecular interaction, potentially disrupted by PRR11 overexpression. f, g HEK293 cells were co-transduced with pLenti7.3-PIK3R1-Flag, -PIK3R1-HA, and pLX302-PRR11-V5 (0, 0.25, 0.5, $1 \mu$ g; f). MCF7 LTED cells that had been stably transduced with pIND-PIK3R1-HA and pLenti6.3-PIK3R1-Flag were transfected with PRR11 siRNA (0, 1, 5, 25 pM) for 48 h in presence of $2 \mu \mathrm{g} / \mathrm{mL}$ doxycycline $(\mathbf{g})$. Cell lysates were precipitated with HA or Flag antibodies and then subjected to immunoblot analysis. $\mathbf{h}$, i MCF7 LTED cells transfected with control or PRR11 siRNA (h) and MDA-MB-175VII cells transduced with pLX302-LacZ or pLX302-PRR11 (i) were serum-starved for 24 $\mathrm{h}$ and then treated with $100 \mathrm{nM}$ insulin for $10 \mathrm{~min}$. Cell lysates were prepared and immunoprecipitated with a p110 $\alpha$ antibody or lgG. Antibody pulldowns were then subjected to immunoblot analysis. j MDA-MB-175VII cells transduced with pLX302-LacZ or pLX302-PRR11 were serum-starved for 24 h and then treated with $100 \mathrm{nM}$ insulin for $10 \mathrm{~min}$. MCF7 LTED and HCC1428 LTED cells transfected with control or PRR11 siRNA were treated with insulin in the same way. Cell lysates were subjected to immunoblot analysis. k HEK293 cells were co-transduced with pLenti7.3-PIK3R1-Flag and either pLX302-PRR11 wild type (WT) or pLX302-PRR11 $\triangle P R$, a mutant lacking the PR motif. Lysates were prepared and immunoprecipitated with a Flag antibody; immune complexes were then subjected to immunoblot analysis. Source data are provided as a Source data file.

significantly abrogated estrogen-independent growth promoted by PRR11 overexpression in MDA-MB-134VI cells (Fig. $7 \mathrm{f}$ and Supplementary Fig. 8e). In addition, treatment with the PI3K inhibitors alpelisib and taselisib abolished estrogen-independent growth of MDA-MB-175VII and MDA-MB-134VI cells stably transduced with PRR11 (Fig. 7g, h). Consistently, PI3K inhibitors led to complete blockade of cyclin D1 protein levels that were induced by PRR11 overexpression (Supplementary Fig. 8f). These data suggest that PRR11-mediated escape from estrogen suppression can be blocked by PI3K inhibition.

\section{Discussion}

The 17 q23 locus is amplified in $\approx 7 \%$ of breast cancers and has been suggested as a molecular subgroup through a clustering analysis of joint copy number and gene expression ${ }^{15}$. This cluster (IntClust1), which is predominantly composed of highly proliferative $\mathrm{ER}^{+}$luminal $\mathrm{B}$ breast cancers, exhibits a poor prognosis and high genomic instability. A recent report showed that IntClust 1 is one of four clusters associated with a high risk of distant relapse of $\mathrm{ER}^{+}$breast cancers in METABRIC ${ }^{16}$. Initial studies aimed at identifying potential oncogenes in 17q23 focused on genes in this locus that are both amplified and overexpressed. RPS6KB1 and TBX2 were first proposed as putative candidates following extensive mapping of the amplicon in breast tumors and breast cancer cell lines ${ }^{44}$. Subsequent comprehensive analysis of copy number and gene expression predicted $M U L, A P P B P 2$, and TRAP240 as potential oncogenes ${ }^{27}$. However, functional studies of these alterations have been incomplete. More recently, WIP1 (PPM1D) and MIR21, also located in 17q23, were shown to cause resistance to anti-HER2 therapy ${ }^{45}$. However, WIP1 and MIR21 were not associated with a worse outcome of patients with $\mathrm{ER}^{+}$breast cancer treated with endocrine therapy in the Kaplan-Meier Plotter dataset (data not shown). In this report, our comprehensive analysis with survival data of patients with ER + breast cancer suggests that PRR11 is strongly associated with breast cancer progression.

Aberrant activation of $\mathrm{PI} 3 \mathrm{~K} / \mathrm{AKT} / \beta$-catenin has been suggested as a mechanism by which PRR11 promotes cell proliferation in ovarian and hepatocellular carcinoma ${ }^{19,46}$, but the molecular basis for a potential role of PRR11 in cancer virulence is unclear. We show herein that the PR domain of PRR11 physically binds the $\mathrm{SH} 3$ domain of $\mathrm{p} 85$, thus inhibiting $\mathrm{p} 85$ homodimer formation. Homodimers or monomers of $\mathrm{p} 85$ form a sequestration complex with IRS1, thus competing with p85-p110 dimers for binding to IRS1 at the plasma membrane and attenuating insulin/ IGF stimulated PI3K activity ${ }^{39}$. Hence, an excess of PRR11 would favor p85-p110 dimers and be permissive of p110 binding to IRS1, retention of p110 at the plasma membrane, and enhanced PIP3 formation. Homodimers of p85 can also inhibit PI3K signaling via direct association and stabilization of $\mathrm{PTEN}^{37}$, but we did not observe that knockdown of PRR11 alters PTEN protein levels (Fig. 5a).

We and others have identified hyperactivation of the PI3K pathway as a mechanism for $\mathrm{ER}^{+}$breast cancers to bypass hormone dependence ${ }^{9,47}$. In this study, we found PRR11 overexpression was strongly associated with resistance to estrogen suppression in primary $\mathrm{ER}^{+}$postmenopausal tumors treated with letrozole. Other analyses shown herein also support a functional connection between PRR11 and the PI3K pathway. For instance, there was a significant correlation between PI3K/IGF1 gene set signature scores and high PRR11 mRNA levels in clinical cohorts of $\mathrm{ER}^{+}$breast tumors treated with neoadjuvant letrozole. Further, PRR11 amplifications were mutually exclusive of PIK3CA mutations in genomic breast cancer databases. Next, overexpression of PRR11 in breast epithelial and breast cancer cells stimulated growth and this effect was abolished by RNA interference of p110 $\alpha$ and by treatment with PI3K inhibitors. Finally, PRR11amplified breast cancer cell lines exhibited higher sensitivity to PI3K inhibitors compared to cells that do not harbor PRR11 amplification. Treatment of patients with advanced $\mathrm{ER}^{+}$breast cancer with PI3Ka inhibitors in combination with antiestrogens has significantly improved progression-free survival ${ }^{13,48}$. In these studies, PIK3CA mutations in tumors predicted clinical benefit from the PI3K inhibitor. However, some patients in these trials with wild type PIK3CA also benefitted clinically, suggesting that other alterations resulting in PI3K pathway dependence also respond to PI3K inhibitors and, as such, should be explored as biomarkers for enrollment of patients into trials with this class of drugs. We posit PRR11 amplification may also serve as a predictive biomarker of sensitivity to $\mathrm{PI} 3 \mathrm{Ka}$ inhibitors, particularly in PIK3CA-wild type tumors.

In summary, we identified PRR11, a gene in the $17 \mathrm{q} 23$ amplicon, as a potential driver of antiestrogen resistance in $\mathrm{ER}^{+}$ breast cancer. Integrative analyses, including clinical data from patients with $\mathrm{ER}^{+}$breast cancer treated with an aromatase inhibitor strongly implicated a role for PRR11 in endocrine resistance. PRR11 blocks p85 homodimerization and sensitizes to ligand-induced PI3K activation, suggesting that PRR11 amplification confers resistance to estrogen deprivation through hyperactivation of the PI3K pathway. Finally, we propose that, in conjunction with endocrine therapy, PI3K may be an actionable target in $\mathrm{ER}^{+}$breast cancers harboring PRR11 amplification. 


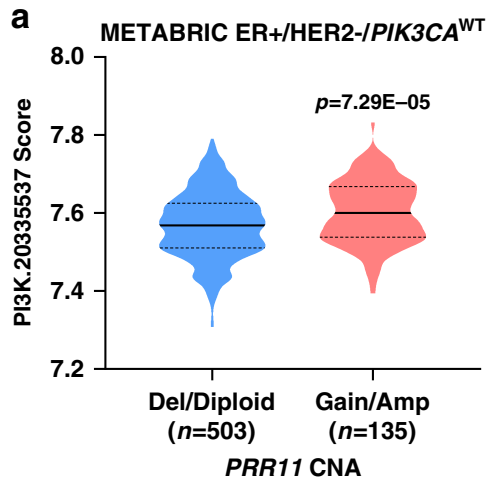

d

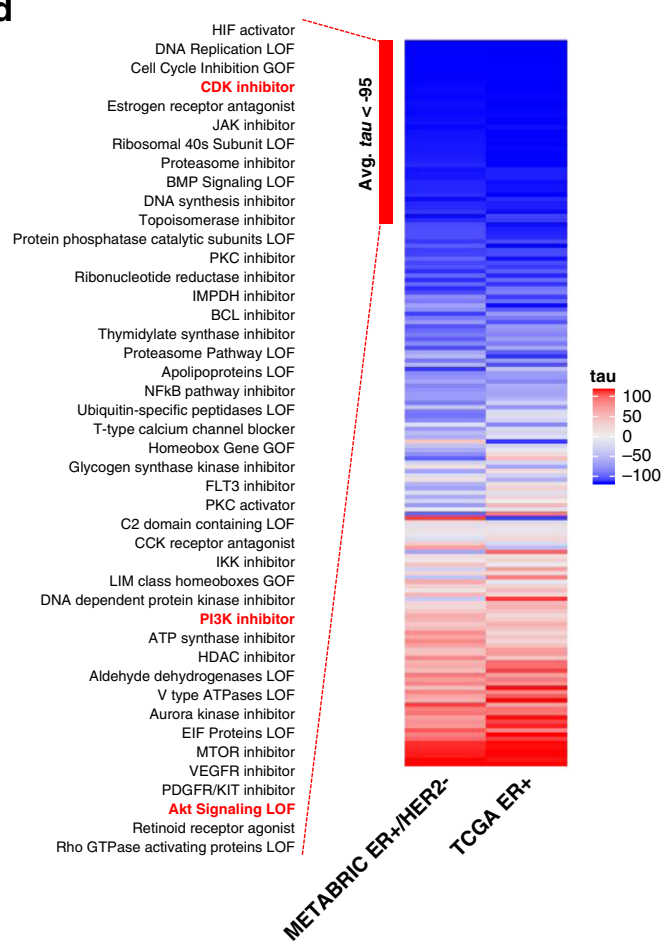

METABRIC ER+/HER2-

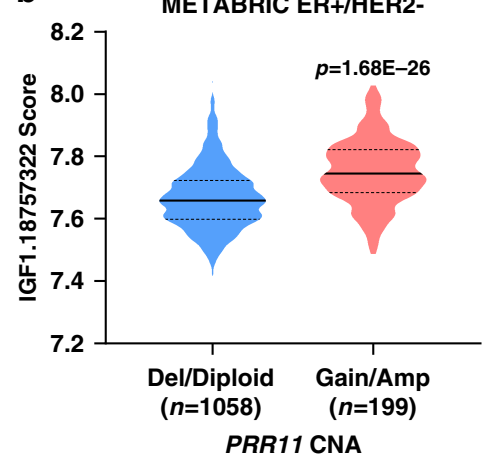

PRR11 CNA
C

METABRIC ER+/HER2-
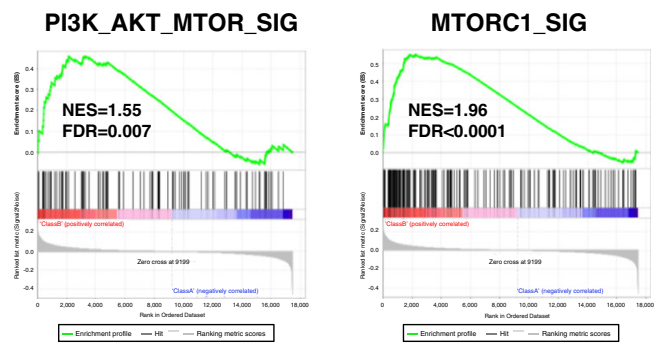

e

METABRIC ER+/HER- $(n=1398)$

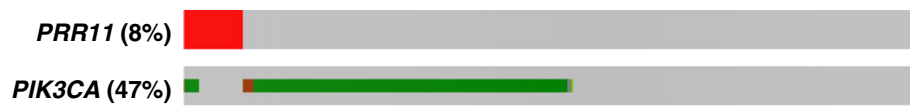

TCGA ER+ $(n=594)$
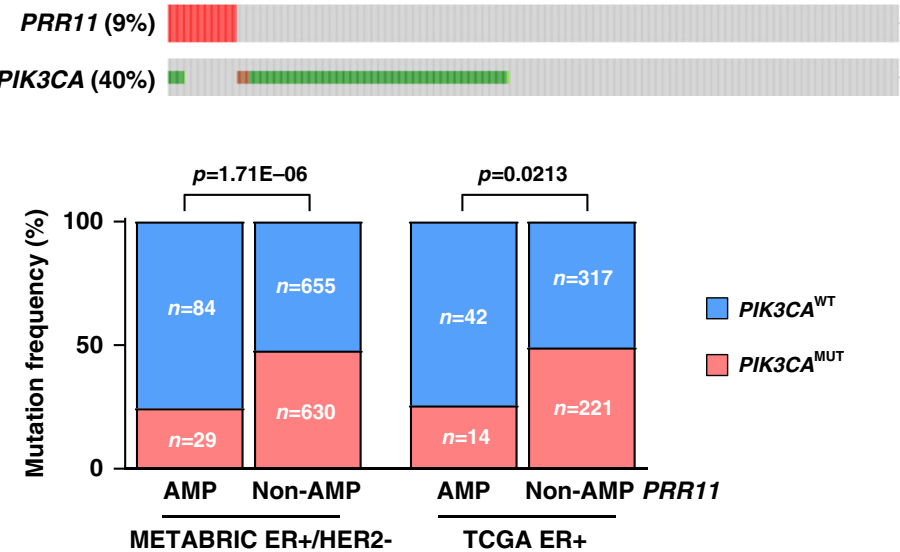

Fig. 6 PRR11 amplification is associated with hyperactivation of the PI3K pathway in ER+ breast cancers. a, b Signature score of the PI3K gene set in ER + /HER2 - /PIK3CA wild type breast cancers in METABRIC plotted as a function of PRR11 copy number (a: $n=503$ and 135 for deletion/diploid and gain/ amplification group, respectively). Signature score of IGF1 gene set in ER+/HER2- breast cancers in METABRIC plotted as a function of PRR11 copy number (b: $n=1058$ and 199 for deletion/diploid and gain/amplification group, respectively). Data represent the mean \pm SD (two-tailed unpaired $t$-tests). c GSEA of mRNA expression data from ER+/HER2- tumors in METABRIC (PRR11 gain/amplification vs deletion/diploid); analyses show enrichment in PI3K_AKT_MTOR_SIG and MTORC1_SIG signatures. d Connectivity scores (tau) were computed using the connectivity map (CMap) with genes significantly upregulated in ER+ (TCGA) breast cancers harboring PRR11 amplification vs. no amplification and ER+/HER2- (METABRIC) breast cancers with PRR11 gain/amplification vs. PRR11 deletion/diploid. Connectivity score of 44 perturbation classes out of 171 are highlighted (tau<-95). e PIK3CA

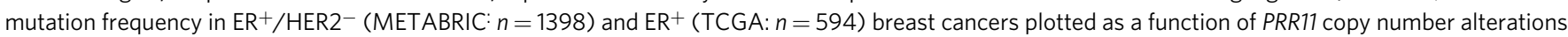
vs. no alterations (two-tailed Fisher's exact test). Source data are provided as a Source data file.

\section{Methods}

Cell lines. The MCF7 (ATCC ${ }^{\oplus}$ HTB-22), HCC1428 (ATCC ${ }^{\circledR}$ CRL-2327), MDA-

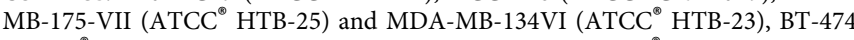
(ATCC ${ }^{\circ}$ HTB-20) human breast cancer cells, HEK293 (ATCC ${ }^{\circ}$ CRL-1573) human embryonic kidney cells and MCF10A (ATCC ${ }^{\oplus}$ CRL-10317) breast epithelial cells were purchased from ATCC in 2018 or 2019. The 293FT (R70007) cells were purchased from Invitrogen in 2016. HCC38 and MDA-MB-231 human breast cancer cells were kindly provided by Dr. Jennifer A. Pietenpol at Vanderbilt University Medical Center. Cell lines were authenticated by the short-tandem repeat (STR) method and tested for Mycoplasma contamination. MCF7, MDAMB-175-VII, MDA-MB-134VI, BT-474, MDA-MB-231, HEK293, and 293FT cells were maintained in DMEM/10\% fetal bovine serum (FBS) $/ 1 \%$ Antibiotic-

Antimycotic (AA). HCC38 and HCC1428 cells were maintained in RPMI 1640/ $10 \% \mathrm{FBS} / 1 \%$ AA. MCF10A cells were maintained in DMEM/F12 supplemented with $5 \%$ horse serum, $20 \mathrm{ng} / \mathrm{mL}$ EGF, $10 \mu \mathrm{g} / \mathrm{mL}$ insulin, $0.5 \mu \mathrm{g} / \mathrm{mL}$ hydrocortisone, $0.1 \mu \mathrm{g} / \mathrm{mL}$ cholera toxin, and 1\% AA. Long-term estrogen-deprived (LTED) cell lines have been described previously ${ }^{9}$. To generate fulvestrant-resistant MCF7 cells, cells were cultured in the presence of increasing concentrations of fulvestrant starting at $50 \mathrm{nM}$. Cells were deemed resistant when they grew as parental cells in 1 $\mu \mathrm{M}$ fulvestrant. To generate tamoxifen-resistant MCF7 and HCC1428 cells, cells were cultured in the presence of increasing concentrations of tamoxifen starting at $500 \mathrm{nM}$. Cells were deemed resistant when they grew as parental cells in $2 \mu \mathrm{M}$ tamoxifen. For experiments outlined here, resistant cells were removed from each drug for at least $24 \mathrm{~h}$ prior to treatment. MCF7 cells (29C-1) whose PIK3CA E545K allele has been corrected to a wild type sequence by somatic cell gene targeting were kindly provided by Dr. Ben Ho Park at Vanderbilt University Medical Center.

Xenograft studies. All mice were maintained according to the guidelines of the Care and Use of Laboratory Animals published by the US National Institutes of Health and the Institutional Animal Care and Use Committee. All procedures were approved by the Institutional Ethics Review Committee of the University of Texas 
a

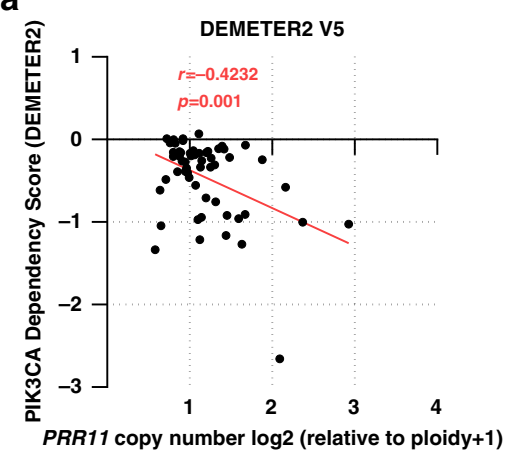

b

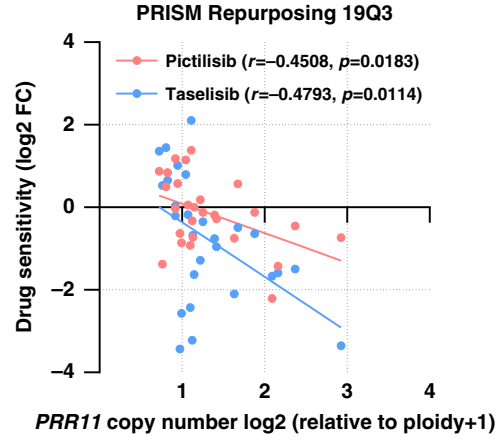

c

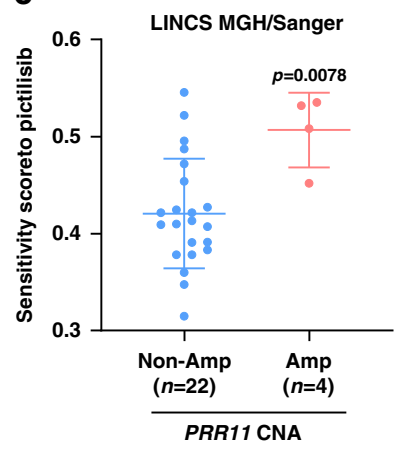

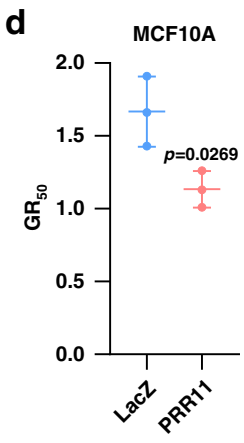

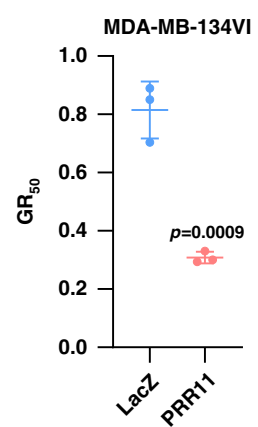

e
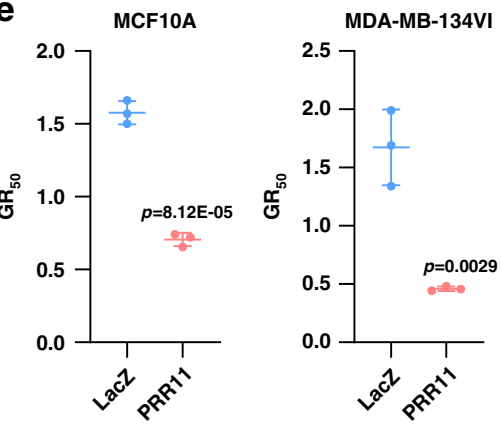

f

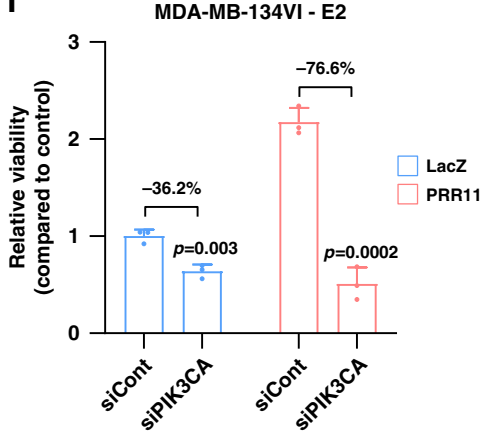

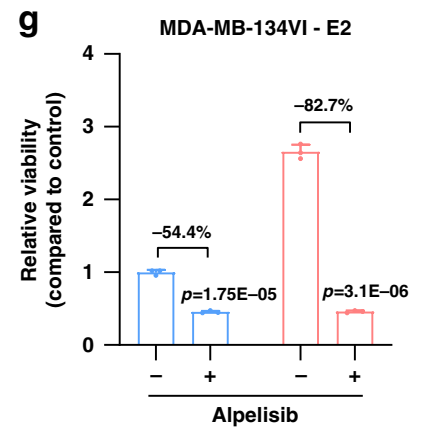
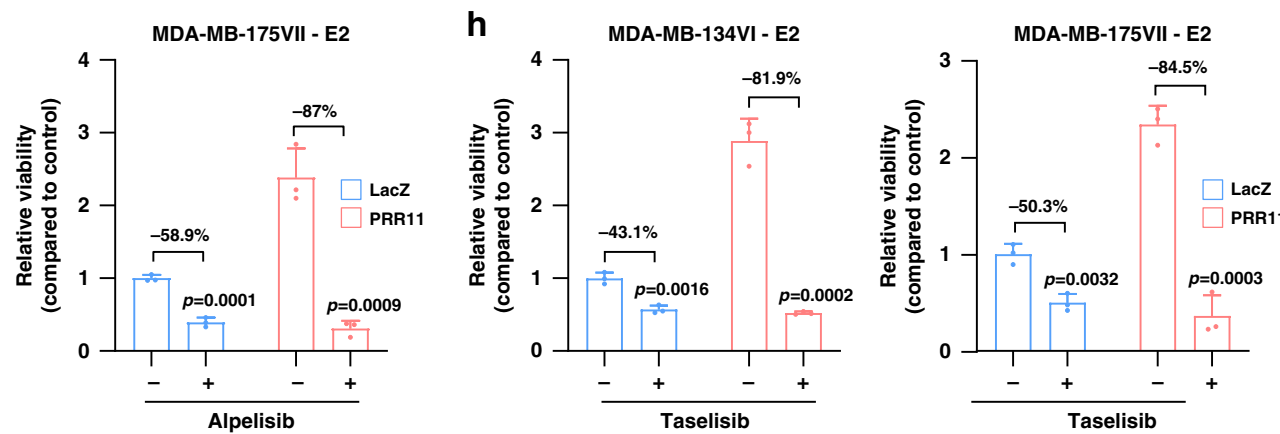

Fig. 7 PRR11-amplified breast cancer cells are dependent on the PI3K pathway. a PIK3CA dependency scores of 57 breast cancer cell lines were plotted against PRR11 copy number (DEMETER2 V5 dataset; Pearson correlation). $\mathbf{b}$ Sensitivity of 27 breast cancer cell lines to pictilisib and taselisib was plotted against PRR11 copy number (PRISM Repurposing 19Q3 dataset; Pearson correlation). Y-axis shows the log 2 cell fraction as per the relative barcode abundance following drug treatment. c Pictilisib sensitivity score of 26 breast cancer cell lines in the LINCS MGH/Sanger dataset $(n=22$ and 4 for nonamplification and amplification group, respectively). Data represent the mean $\pm S D$ (two-tailed unpaired $t$-tests). d, e Alpelisib (d) and taselisib (e) $G_{50}$ of MDA-MB-134VI and MDA-MB-175VII cells transduced with pLX302-LacZ or pLX302-PRR11 were calculated using the GR metrics calculator. Cell numbers counted at day 0 and day 6 were used as the input data. Data represent the mean $\pm S D$ of three replicates (two-tailed unpaired $t$-tests). $\mathbf{f}$ Low density monolayers of MDA-MB-134VI pLX302-LacZ and -PRR11 cells were grown in estrogen (E2)-free medium. After 14 days, cell monolayers were stained with crystal violet. Data represent the mean \pm SD of three replicates (two-tailed unpaired $t$-tests). $\mathbf{g}, \mathbf{h}$ Low density monolayers of MDA-MB-175VII and MDAMB-134VI cells transduced with pLX302-PRR11 or pLX302-LacZ were treated $\pm 1 \mu \mathrm{M}$ alpelisib (g) or $\pm 1 \mu \mathrm{M}$ taselisib (h) in estrogen-free medium. After 14 days, cell monolayers were stained with crystal violet. Data represent the mean \pm SD of three replicates (two-tailed unpaired $t$-tests). Source data are provided as a Source data file.

Southwestern Medical Center. Eight-week old female ovariectomized athymic mice (Hsd:Athymic Nude-Foxn $1^{\text {nu }}$, Envigo) were implanted with a 14-day release $17 \beta-$ estradiol pellet $\left(0.17 \mathrm{mg}\right.$, Innovative Research of America). The following day, $10^{7}$ MCF7 cells stably expressing a doxycycline-inducible control or PRR11 shRNA were suspended in IMEM and growth factor reduced Matrigel (BD Biosciences) at a 1:1 ratio and then injected subcutaneously into the right flank of each mouse. Approximately 4 weeks later, mice bearing tumors measuring $\geq 250 \mathrm{~mm}^{3}$ were randomized to treatment with vehicle $(0.9 \% \mathrm{NaCl})$ or doxycycline $(10 \mathrm{mg} / \mathrm{kg} / \mathrm{daily}$, by intraperitoneal injection). Tumor diameters were measured with calipers weekly and tumor volume was calculated with the formula: volume $=$ width $^{2} \times$ length $/ 2$. After 4 weeks, tumors were harvested and homogenized using TissueLyser II (Qiagen) for subsequent immunoblot analysis.

Gene set signature analyses. Single-sample gene set enrichment for 125 previously published breast cancer-related gene expression signatures were computed as described previously ${ }^{17}$; signatures with false discovery rate (FDR) $<0.01$ were considered as differentially activated pathways between PRR11 high and low tumors. Gene set enrichment analysis (GSEA) was conducted with the javaGSEA interface downloaded from Broad Institute (http://software.broadinstitute.org/gsea/ index.jsp). The h.all.v6.2.ymbols.gmt [Hallmarks] was used as gene sets database ${ }^{49}$.

Immunohistochemistry (IHC). Formalin-fixed paraffin-embedded (FFPE) 4- $\mu \mathrm{m}$ tumor sections were deparaffinized. Antigen retrieval was performed with a citrate buffer ( $\mathrm{pH}$ 6) in a decloaking chamber (Biocare). Endogen peroxidase was blocked with $3 \% \mathrm{H}_{2} \mathrm{O}_{2}$ and protein block (Agilent). Tumor sections were next incubated with a PRR11 antibody (Novus, NBP1-83784; dilution 1:200) overnight at $4^{\circ} \mathrm{C}$. Envision (Agilent) was used for visualization with DAB as the chromogen (Agilent); hematoxylin was applied as the counterstain. Whole sections were digitally acquired using an AxioScan Z1 slide scanner (Carl Zeiss) at $\times 20$. Automated semiquantitative scoring was performed using QuPath software ${ }^{50}$. Color deconvolution 
stains were set form a representative area. A cell segmentation was determined on hematoxylin OD. An object classification was trained to differentiate tumor from stroma. Percentage of PRR11 ${ }^{+}$cells was calculated with the cell detection algorithm according to the cytoplasm DAB OD mean. Each selected region was visually assessed for correct performance of the quantification algorithm excluding areas of non-invasive tumor.

Fluorescence in situ hybridization. Four- $\mu \mathrm{m}$ tissue sections were mounted on charged slides and hybridized overnight with a PRR11 FISH probe (EMPIRE GENOMICS) and a centromere 17 control probe (EMPIRE GENOMICS); FISH was performed as described previously ${ }^{14}$. Twenty to sixty tumor cell nuclei from random areas were individually evaluated with the $100 \times$ oil immersion objective by counting green PRR11 and orange centromere 17 (CEN17) signals. A PRR11/ CEN17 ratio $\geq 2.0$ was considered as $P R R 11$ amplification.

siRNA transfection. Cells seeded in 6-well plates or 60 -mm dishes were transfected with 20 pmole or 40 pmole of siRNAs, respectively, using Lipofectamine RNAiMAX reagent (Invitrogen) as per the manufacturer's instructions. Control siRNA (4390843), PRR11 siRNAs (siRNA\#1: 4392420-S31473, siRNA\#2: 4392420S31475), and PIK3CA siRNA (4390824-S10520) were purchased from ThermoFisher Scientific.

Plasmids. Human PRR11 open reading frame (ORF) in pENRT221 from The Ultimate $^{\mathrm{TM}}$ ORF Lite human cDNA collection (Life Technologies) was cloned into pLX302 or pINDUCER20 (pIND) using Gateway ${ }^{\text {TM }}$ LR Clonase ${ }^{\text {TM }}$ II Enzyme Mix (ThermoFisher). To generate pLX302-PRR11- $\triangle \mathrm{PR}$, the PR motif was predicted using Motif Scan (https://myhits.isb-sib.ch/cgi-bin/motif_scan) and then removed from the pLX302-PRR11-WT construct using Q5-Site-Directed Mutagenesis Kit (NEW ENGLAND BioLabs). pLX304-PRR11- $\triangle \mathrm{PR}$ was generated from pLX302PRR11- $\triangle$ PR through BP/LR cloning. SMART doxycycline-inducible PRR11 shRNA and SMART PRR11 shRNA targeting 3'UTR was purchased from Dharmacon (V3SH11252 and V3SH11240, respectively). pLenti7.3-PIK3R1-Flag and pLenti7.3PIK3R1-HA were kindly provided by Dr. Gordon Mills at Oregon Health Sciences University. To generate pLenti6.3-PIK3R1-Flag and pIND-PIK3R1-HA, PIK3R1Flag and PIK3R1-HA were cloned into pDORN ${ }^{\mathrm{TM}} 221$ using Gateway ${ }^{\mathrm{TM}}$ BP Clonase ${ }^{\mathrm{TM}}$ II Enzyme mix (ThermoFisher). Next, pDONR221-PIK3R1-Flag and pDONR221-PIK3R1-HA were cloned into pLenti6.3/V5-DSET ${ }^{\mathrm{TM}}$ Gateway $^{\mathrm{TM}}$ vector (ThermoFisher) and pINDUCER20, respectively, using Gateway ${ }^{\mathrm{TM}}$ LR Clonase $^{\mathrm{TM}}$ II Enzyme Mix. pLX304-PRR11(HsCD00444919), -BRIP1 (HsCD00440250), -SMARCD2 (HsCD00440288) and -TACO1 (HsCD00442382) were purchased from DNASU.

Lentiviral transduction. To generate stably transduced cell lines, $1 \mu \mathrm{g}$ of the plasmids were co-transfected with $0.75 \mu \mathrm{g}$ of psPAX2 (2nd generation lentiviral packaging plasmid) and $0.5 \mu \mathrm{g}$ of pMD2.G (VSV-G envelope expressing plasmid) into 293FT cells using Lipofectamine2000 (ThermoFisher). Cell medium was changed to fresh medium $24 \mathrm{~h}$ post-transfection, and cells were collected $48 \mathrm{~h}$ later. Lentiviral particles of sgRNA editing PRR11 were purchased from Dharmacon (VSGH11937-247492131). Virus-containing medium was applied to target cells with $8 \mu \mathrm{g} / \mathrm{mL}$ polybrene. Puromycin were used for selection of cells transduced with SMART doxycycline-inducible PRR11 shRNA, SMART PRR11 shRNA targeting 3' UTR, pLX302-PRR11, pLX302-LacZ, control sgRNA and PRR11 sgRNA. Blasticidin were used for selection of cells transduced with pLX304-GFP, -PRR11, -BRIP1, -SMARCD2, -TACO1 or pLenti6.3-PIK3R1-Flag. MCF7 LTED cells transduced with pIND-PIK3R1-HA were selected with G418 sulfate.

Cell proliferation assays. After $24 \mathrm{~h}$ from transfection with control siRNA or PRR11 siRNAs, HCC1428, and MCF7 (LTED, TamR, and FulvR) cells were seeded in 6-well plates. Cells were trypsinized and then counted every 3 days for 6 days. Number of cells were counted with Z2 coulter counter analyzer (Beckman coulter).

RT-qPCR. RNA was extracted from cells using Maxwell RSC simplyRNA Cells Kit (Promega) according to the manufacturer's protocol. cDNA was synthesized using the iSCRIPT cDNA synthesis Kit (Bio-Rad) and then subjected to PCR with PowerUp ${ }^{\text {TM }}$ SYBR $^{\text {TM }}$ Green Master Mix (ThermoFisher), PIK3CA (Qiagen), or GAPDH (Qiagen) primers using QuantStudio3 Real-Time PCR System (ThermoFisher)

Dual luciferase assay. Cells $\left(1 \times 10^{4} /\right.$ well $)$ were seeded in 96 -well plates in triplicate. Next day, cells were transfected with pGLB-MERE and pCMV-Renilla, each with either control or PRR11 siRNA. Twenty-four h post-transfection, cells were switched to estrogen-free medium (IMEM/5\% charcoal stripped FBS) for $24 \mathrm{~h}$, followed by treatment with $1 \mathrm{nM}$ estradiol for $24 \mathrm{~h}$. Renilla and firefly luciferase activities were measured using Dual-Luciferase ${ }^{\circledR}$ Reporter Assay System according to manufacturer's protocol (Promega).
Clonogenic assays. MCF7 and HCC1428 (LTED, TamR, and FulvR) cells were seed in 12-well plate and then transfected with control siRNA or PRR11 siRNA. Cells were grown $\pm 1 \mathrm{nM}$ estradiol, $\pm 1 \mu \mathrm{M}$ tamoxifen or $\pm 1 \mu \mathrm{M}$ fulvestrant for 10 days. MDA-MB-175VII cells transduced with pLX304-PRR11, -BRIP1, -SMARCD2 and -TACO1 were grown in absence of estradiol for 14 days. MDAMB-175VII and MDA-MB-134VI cells transduced with pLX302-PRR11 or -LacZ were grown in absence of estradiol $\pm 1 \mu \mathrm{M}$ taselisib or $\pm 1 \mu \mathrm{M}$ alpelisib for 14 days. MCF10A pLX302-PRR11 or - LacZ cells were grown $\pm 1 \mu \mathrm{M}$ taselisib or $\pm 1 \mu \mathrm{M}$ alpelisib for 10 days. Fresh media containing drugs were replaced every 4 days. Cells were fixed with cold-iced methanol for $10 \mathrm{~min}$ at $-20{ }^{\circ} \mathrm{C}$ and then stained with $0.5 \%$ crystal violet solution for $10 \mathrm{~min}$ at room temperature. Stained monolayers were imaged using Gelcount mammalian cell colony counter (Oxford Optronix). For quantification, cell monolayers were dissolved with $10 \%$ acetic acid for $15 \mathrm{~min}$ at room temperature. Supernatants were transferred into 96-well plates for measuring absorbance at $560 \mathrm{~nm}$ using GloMax Discover microplate reader (Promega).

Immunoblot analysis. Cells were lysed with RIPA buffer (ThermoFisher) containing protease inhibitors (Protease Inhibitor Cocktail, Roche) and phosphatase inhibitors (PhosSTOP, Roche), scraped and then incubated on ice for $30 \mathrm{~min}$. Supernatants were collected after centrifugation at $14,000 \mathrm{rpm}$ for $10 \mathrm{~min}$. Protein concentration in cell lysates was measured with the Pierce BCA Protein Assay kit (ThermoFisher). Thirty $\mu \mathrm{g}$ of protein were subjected to SDS-PAGE followed by transfer to nitrocellulose membrane for immunoblot analysis. Information of antibodies used in this study is included as Supplementary Table 6.

Cell cycle analysis. After $48 \mathrm{~h}$ from transfection with control siRNA or PRR11 siRNAs, cells were trypsinized and then fixed with $70 \%$ ethanol for $3 \mathrm{~h}$ at $-20{ }^{\circ} \mathrm{C}$. Cells were washed x 3 with PBS and then resuspended in PBS containing $100 \mu \mathrm{g} / \mathrm{mL}$ RNase A and $40 \mu \mathrm{g} / \mathrm{mL}$ propidium iodide for $10 \mathrm{~min}$ at room temperature. Stained cells were analyzed with BD LSRFORTESSA (BD Biosciences) followed by profiling of cell cycle phases with the Dean-Jett-Fox model of FlowJo (ver.10). Gating strategy is shown in Supplementary Fig. $4 \mathrm{i}$.

PCR array. RNA was extracted from MCF7 LTED cells transfected with control siRNA or PRR11 siRNA for $48 \mathrm{~h}$ using Maxwell ${ }^{\circledast}$ RSC simplyRNA Cells Kit (Promega). Complementary DNA (cDNA) was synthesized using RT2 First Strand Kit according to the manufacturer's protocol and then subjected to real-time PCR using the RT ${ }^{2}$ Profiler ${ }^{\mathrm{TM}}$ PCR Array Human Cell Cycle (Qiagen) in duplicate.

Live cell imaging. A plasmid encoding the GFP-based AKT-PH biosensor was designed as described in a previous report ${ }^{51}$. Briefly, the PH domain of AKT1 (residues 2-149) was fused with the COOH terminus of enhanced GFP. MCF7 LTED cells were transduced with pEGFP-AKT-PH and then sorted for GFP expression by flow cytometry. For three-dimensional imaging, we used a modified variant of the light-sheet microscope ${ }^{52}$. Briefly, this microscope illuminates the specimen from the epi-direction with an obliquely launched light-sheet. The beam is rapidly scanned, and subsequent fluorescence descanned, using a mirror galvanometer. For primary, secondary, and tertiary objectives, we used a high-NA silicone immersion objective (Nikon $\times 100$ NA $1.35,0.28-0.31 \mathrm{~mm}$ working distance), a high-NA air immersion objective (Nikon $\times 40$ NA $0.95,0.25-0.16 \mathrm{~mm}$ working distance), and a bespoke glass-tipped objective (AMS-AGY v1.0, NA 1.0, $0 \mathrm{~mm}$ working distance. https://andrewgyork.github.io/

high_na_single_objective_lightsheet/), respectively. Images were acquired for each plane on a high-speed scientific CMOS camera (Hamamatsu Flash 4.0) using custom LabView-based software, sheared in the frequency-domain with Python, and rotated with an affine transform using either MATLAB or IMOD. No deconvolution was performed on the data shown. For each condition, 8 positions in a $35 \mathrm{~mm}$ dish were chosen at random, and each position encompassed multiple cells. Movies were acquired at a volumetric imaging rate of $0.1 \mathrm{~Hz}$ for $10 \mathrm{~min}$.

Immunoprecipitation. All immunoprecipitation experiments were performed using the Dynabeads ${ }^{\mathrm{TM}}$ Protein G Immunoprecipitation Kit (ThermoFisher) according to the manufacturer's protocol. Briefly, $10 \mu \mathrm{g}$ of antibodies were preincubated with $1.5 \mathrm{mg}$ Dynabeads for $10 \mathrm{~min}$ at room temperature and then incubated overnight at $4{ }^{\circ} \mathrm{C}$ with $1 \mathrm{mg}$ of cell lysate. Precipitates were eluted from the magnetic bead-antibody-antigen complex using the elution buffer containing NuPAGE LDS sample buffer (ThermoFisher) and NuPAGE sample reducing agent (ThermoFisher) for $10 \mathrm{~min}$ at $70^{\circ} \mathrm{C}$. Eluted samples and $20 \mu \mathrm{g}$ of input lysates were subjected to immunoblot analysis.

Proximity ligation assay. MCF7 LTED $\left(5 \times 10^{4} /\right.$ well $)$ cells were seeded in 8 -well chamber slides (Lab-Tek, 177445) in triplicate. HEK293 cells $\left(5 \times 10^{4}\right)$ were seeded $24 \mathrm{~h}$ post-transfection with pLX302-PRR11-V5, pLenti7.3-PIK3R1-Flag, and pLenti7.3-PIK3R1-HA. PRR11 (Cat\# LS-B15222, LS Bio). p85 (Cat\# 05-212, Millipore), V5 (Cat\# 13202, Cell Signaling Technology), HA (Cat\# 3724, Cell Signaling Technology) and Flag (Cat\# 8146, Cell Signaling Technology) antibodies were used for this assay. PLA was performed with Duolink In Situ Red Starter Kit Mouse/ 
Rabbit (Sigma) according to the manufacturer's protocol and then imaged with a DMi8 inverted microscope (Leica). The number of PLA foci was quantified by Duolink Image Tool software; 5 images per sample were analyzed.

Statistics and reproducibility. Pearson $r$ correlation, hazard ratio and $t$-tests (Nonparametric tests) were performed with GraphPad Prism version 8 or Microsoft Excel 2016. Data were represented as mean \pm SD. All experiments were conducted at least three times. A $p$ value $<0.05$ was considered statistically significant. A false discovery rate (FDR) was computed using the Benjamini-Hochberg procedure. $\mathrm{R}$ version 3.5.2 and $\mathrm{R}$ studio version 1.1.463 were used.

Reporting summary. Further information on research design is available in the Nature Research Reporting Summary linked to this article.

\section{Data availability}

All data associated with this study are present in the paper or Supplementary information. Raw RNA sequencing and clinical data of $\mathrm{ER}^{+}$breast tumors following long-term neoadjuvant treatment with letrozole were obtained from the previous report ${ }^{17}$ and are available in the Sequence Read Archive under Bioproject accession code PRJNA605185 and have been deposited in the Gene Expression Omnibus under accession code GSE145325. RNA-seq (fragments per kilobase million; FPKM) and breast tumor Ki67 data of the cohort of Miller et al. ${ }^{22}$ were downloaded from Supplementary information of the corresponding article. Raw RNA-seq data of the cohort of Giltnane et al. ${ }^{14}$ was obtained from the Sequence Read Archive under Bioproject accession code PRJNA272565. These published clinical cohorts are summarized in Supplementary Table 1. Somatic mutations, normalized gene expression, and clinical data in The Cancer Genome Atlas (TCGA; Cell 2015 53 ), METABRIC (Nature 2012 and Nat Commun $2016^{54}$ ) were downloaded from cBioPortal ${ }^{55}$. Copy number aberration and mutation data of metastatic breast cancers were obtained from The Metastatic Breast Cancer Project (https://www.mbcproject.org/), a project of Count Me In (https://joincountmein.org/). Gene expression, copy number, dependence and drug sensitivity data of breast cancer cell lines were downloaded through the DepMap portal (https://depmap.org/portal/). The code for 125 breast cancer-related signature is available at https:/github.com/kmlee1982/ Arteaga_lab. Source data are provided with this paper.

\section{Code availability}

The code for 125 breast cancer-related signature is available at https://github.com/ kmlee1982/Arteaga_lab.

Received: 19 March 2020; Accepted: 6 October 2020;

Published online: 30 October 2020

\section{References}

1. Cancer Genome Atlas, N. Comprehensive molecular portraits of human breast tumours. Nature 490, 61-70 (2012).

2. Howell, A. et al. Results of the ATAC (Arimidex, Tamoxifen, Alone or in Combination) trial after completion of 5 years' adjuvant treatment for breast cancer. Lancet 365, 60-62 (2005).

3. Early Breast Cancer Trialists' Collaborative, G. Aromatase inhibitors versus tamoxifen in early breast cancer: patient-level meta-analysis of the randomised trials. Lancet 386, 1341-1352 (2015).

4. Ma, C. X., Reinert, T., Chmielewska, I. \& Ellis, M. J. Mechanisms of aromatase inhibitor resistance. Nat. Rev. Cancer 15, 261-275 (2015).

5. Jeselsohn, R. et al. Emergence of constitutively active estrogen receptor-alpha mutations in pretreated advanced estrogen receptor-positive breast cancer. Clin. Cancer Res. 20, 1757-1767 (2014).

6. Arpino, G. et al. HER-2 amplification, HER-1 expression, and tamoxifen response in estrogen receptor-positive metastatic breast cancer: a southwest oncology group study. Clin. Cancer Res. 10, 5670-5676 (2004).

7. Formisano, L. et al. Association of FGFR1 with ERalpha maintains ligandindependent ER transcription and mediates resistance to estrogen deprivation in ER(+) breast cancer. Clin. Cancer Res. 23, 6138-6150 (2017).

8. Razavi, P. et al. The genomic landscape of endocrine-resistant advanced breast cancers. Cancer Cell 34, 427-438 (2018). e426.

9. Miller, T. W. et al. Hyperactivation of phosphatidylinositol-3 kinase promotes escape from hormone dependence in estrogen receptor-positive human breast cancer. J. Clin. Invest. 120, 2406-2413 (2010).

10. Sanchez, C. G. et al. Preclinical modeling of combined phosphatidylinositol-3kinase inhibition with endocrine therapy for estrogen receptor-positive breast cancer. Breast Cancer Res. 13, R21 (2011).
11. Juric, D. et al. Alpelisib plus fulvestrant in PIK3CA-Altered and PIK3CAWild-type Estrogen Receptor-positive Advanced Breast Cancer: A Phase $1 \mathrm{~b}$ clinical trial. JAMA Oncol. 5, e184475 (2018).

12. Dickler, M. N. et al. Phase II study of taselisib (GDC-0032) in combination with fulvestrant in patients with HER2-negative, hormone receptor-positive advanced breast cancer. Clin. Cancer Res. 24, 4380-4387 (2018).

13. Andre, F. et al. Alpelisib for PIK3CA-mutated, hormone receptor-positive advanced breast cancer. N. Engl. J. Med. 380, 1929-1940 (2019).

14. Giltnane, J. M. et al. Genomic profiling of ER $(+)$ breast cancers after shortterm estrogen suppression reveals alterations associated with endocrine resistance. Sci. Transl. Med. 9, eaai7993 (2017).

15. Dawson, S. J., Rueda, O. M., Aparicio, S. \& Caldas, C. A new genome-driven integrated classification of breast cancer and its implications. EMBO J. 32, 617-628 (2013).

16. Rueda, O. M. et al. Dynamics of breast-cancer relapse reveal late-recurring ER-positive genomic subgroups. Nature 567, 399-404 (2019).

17. Guerrero-Zotano, A. L. et al. ER(+) breast cancers resistant to prolonged neoadjuvant letrozole exhibit an E2F4 transcriptional program sensitive to CDK4/6 inhibitors. Clin. Cancer Res. 24, 2517-2529 (2018).

18. Ji, Y. et al. PRR11 is a novel gene implicated in cell cycle progression and lung cancer. Int J. Biochem Cell Biol. 45, 645-656 (2013).

19. Zhu, J., Hu, H., Wang, J., Yang, Y. \& Yi, P. PRR11 Overexpression facilitates ovarian carcinoma cell proliferation, migration, and invasion through activation of the PI3K/AKT/beta-catenin pathway. Cell Physiol. Biochem. 49, 696-705 (2018).

20. Song, Z. et al. PRR11 Is a prognostic marker and potential oncogene in patients with gastric cancer. PLoS ONE 10, e0128943 (2015).

21. Gyorffy, B. et al. An online survival analysis tool to rapidly assess the effect of 22,277 genes on breast cancer prognosis using microarray data of 1,809 patients. Breast Cancer Res. Treat. 123, 725-731 (2010).

22. Miller, C. A. et al. Aromatase inhibition remodels the clonal architecture of estrogen-receptor-positive breast cancers. Nat. Commun. 7, 12498 (2016).

23. Dutertre, M. et al. Estrogen regulation and physiopathologic significance of alternative promoters in breast cancer. Cancer Res. 70, 3760-3770 (2010).

24. Ghandi, M. et al. Next-generation characterization of the cancer cell line encyclopedia. Nature 569, 503-508 (2019).

25. Huret, J. L. et al. Atlas of genetics and cytogenetics in oncology and haematology in 2013. Nucleic Acids Res. 41, D920-D924 (2013).

26. Kelemen, L. E. et al. Genetic variation in the chromosome 17 q23 amplicon and breast cancer risk. Cancer Epidemiol. Biomark. Prev. 18, 1864-1868 (2009).

27. Monni, O. et al. Comprehensive copy number and gene expression profiling of the 17q23 amplicon in human breast cancer. Proc. Natl Acad. Sci. USA 98, 5711-5716 (2001)

28. Cowley, G. S. et al. Parallel genome-scale loss of function screens in 216 cancer cell lines for the identification of context-specific genetic dependencies. Sci. Data 1, 140035 (2014).

29. Shao, D. D. et al. ATARiS: computational quantification of gene suppression phenotypes from multisample RNAi screens. Genome Res. 23, 665-678 (2013).

30. Corsello, S. M. et al. Discovering the anti-cancer potential of non-oncology drugs by systematic viability profiling. Nat. Cancer1, 235-248 (2020).

31. Stover, D. G. et al. The role of proliferation in determining response to neoadjuvant chemotherapy in breast cancer: a gene expression-based metaanalysis. Clin. Cancer Res. 22, 6039-6050 (2016).

32. Fox, E. M. et al. A kinome-wide screen identifies the insulin/IGF-I receptor pathway as a mechanism of escape from hormone dependence in breast cancer. Cancer Res 71, 6773-6784 (2011).

33. Cross, D. A., Alessi, D. R., Cohen, P., Andjelkovich, M. \& Hemmings, B. A. Inhibition of glycogen synthase kinase- 3 by insulin mediated by protein kinase B. Nature 378, 785-789 (1995).

34. Lawlor, M. A. \& Alessi, D. R. PKB/Akt: a key mediator of cell proliferation, survival and insulin responses? J. Cell Sci. 114, 2903-2910 (2001).

35. Zarrinpar, A., Bhattacharyya, R. P. \& Lim, W. A. The structure and function of proline recognition domains. Sci. STKE 2003, RE8, https://doi.org/10.1126/ stke.2003.179.re8 (2003)

36. Yu, J. et al. Regulation of the p85/p110 phosphatidylinositol 3'-kinase: stabilization and inhibition of the p110alpha catalytic subunit by the p 85 regulatory subunit. Mol. Cell Biol. 18, 1379-1387 (1998).

37. Cheung, L. W. et al. Regulation of the PI3K pathway through a p85alpha monomer-homodimer equilibrium. Elife 4, e06866 (2015).

38. Harpur, A. G. et al. Intermolecular interactions of the p85alpha regulatory subunit of phosphatidylinositol 3-kinase. J. Biol. Chem. 274, 12323-12332 (1999).

39. Luo, J., Field, S. J., Lee, J. Y., Engelman, J. A. \& Cantley, L. C. The p85 regulatory subunit of phosphoinositide 3-kinase down-regulates IRS-1 signaling via the formation of a sequestration complex. J. Cell Biol. 170, 455-464 (2005). 
40. Lamb, J. et al. The Connectivity Map: using gene-expression signatures to connect small molecules, genes, and disease. Science 313, 1929-1935 (2006).

41. McFarland, J. M. et al. Improved estimation of cancer dependencies from large-scale RNAi screens using model-based normalization and data integration. Nat. Commun. 9, 4610 (2018).

42. Duan, Q. et al. Drug/cell-line browser: interactive canvas visualization of cancer drug/cell-line viability assay datasets. Bioinformatics 30, 3289-3290 (2014).

43. Dogruluk, T. et al. Identification of variant-specific functions of PIK3CA by Rapid Phenotyping of Rare Mutations. Cancer Res 75, 5341-5354 (2015).

44. Sinclair, C. S., Rowley, M., Naderi, A. \& Couch, F. J. The 17 q23 amplicon and breast cancer. Breast Cancer Res Treat. 78, 313-322 (2003).

45. Liu, Y. et al. Targeting $17 \mathrm{q} 23$ amplicon to overcome the resistance to antiHER2 therapy in HER2+ breast cancer. Nat. Commun. 9, 4718 (2018).

46. Qiao, W., Wang, H., Zhang, X. \& Luo, K. Proline-rich protein 11 silencing inhibits hepatocellular carcinoma growth and epithelial-mesenchymal transition through beta-catenin signaling. Gene 681, 7-14 (2019).

47. Campbell, R. A. et al. Phosphatidylinositol 3-kinase/AKT-mediated activation of estrogen receptor alpha: a new model for anti-estrogen resistance. J. Biol. Chem. 276, 9817-9824 (2001).

48. Saura, C. et al. Neoadjuvant letrozole plus taselisib versus letrozole plus placebo in postmenopausal women with oestrogen receptor-positive, HER2negative, early-stage breast cancer (LORELEI): a multicentre, randomised, double-blind, placebo-controlled, phase 2 trial. Lancet Oncol. 20, 1226-1238 (2019).

49. Subramanian, A. et al. Gene set enrichment analysis: a knowledge-based approach for interpreting genome-wide expression profiles. Proc. Natl Acad. Sci. USA 102, 15545-15550 (2005).

50. Bankhead, P. et al. QuPath: Open source software for digital pathology image analysis. Sci. Rep. 7, 16878 (2017).

51. Haugh, J. M., Codazzi, F., Teruel, M. \& Meyer, T. Spatial sensing in fibroblasts mediated by 3' phosphoinositides. J. Cell Biol. 151, 1269-1280 (2000)

52. Yang, B. et al. Epi-illumination SPIM for volumetric imaging with high spatial-temporal resolution. Nat. Methods 16, 501-504 (2019).

53. Ciriello, G. et al. Comprehensive molecular portraits of invasive lobular breast cancer. Cell 163, 506-519 (2015).

54. Pereira, B. et al. The somatic mutation profiles of 2,433 breast cancers refines their genomic and transcriptomic landscapes. Nat. Commun. 7, 11479 (2016).

55. Gao, J. et al. Integrative analysis of complex cancer genomics and clinical profiles using the cBioPortal. Sci. Signal 6, pll (2013).

56. Hafner, M., Niepel, M., Chung, M. \& Sorger, P. K. Growth rate inhibition metrics correct for confounders in measuring sensitivity to cancer drugs. Nat. Methods 13, 521-527 (2016).

\section{Acknowledgements}

This study was supported by NCI Breast SPORE grant P50 CA098131, VanderbiltIngram Cancer Center P30 CA68485, UTSW Simmons Cancer Center P30 CA142543, CPRIT RR170061 grant (C.L.A.), Susan G. Komen Breast Cancer Foundation grant SAC100013 (C.L.A.), grants from the Breast Cancer Research Foundation (C.L.A., L.C. C.), Susan G. Komen Postdoctoral Fellowship PDF17487926 (K.-M.L.), NIH grants R35 CA197588 (L.C.C.), U54 U54CA210184 (L.C.C.), and the Gray Foundation (L.C.C.). We thank Dr. Jennifer A. Pietenpol, Dr. Ben Ho Park, and Dr. Gordon Mills for providing cell lines and materials.

\section{Author contributions}

K.-M.L., A.L.G.-Z., L.C.C., and C.L.A. designed this study. K.-M.L., A.L.G.-Z., V.M.J., and K.D. contributed to development of methodology. K.-M.L., C.C.L., P. G.-E., M.E.S., T.P. S., G.R., K.D., and R.F. contributed to acquisition of data. K.-M.L., A.B.H., A.S., D.R.S., L. F., K.D., and C.L.A. analyzed and interpreted data. K.-M.L., A.B.H., and C.L.A. wrote the paper.

\section{Competing interests}

C.L. Arteaga has received research grants from Pfizer, Lilly, and Takeda. He holds minor stock options in Provista and Y-TRAP, and serves or has served in an advisory role to Novartis, Merck, Lilly, Daiichi Sankyo, Taiho Oncology, OrigiMed, Puma Biotechnology, Immunomedics, AstraZeneca, and Sanofi. He is a member of the Scientific Advisory Board (SAB) of the Susan G. Komen Foundation. L.C. Cantley is a founder and member of the SAB and holds equity in Agios Pharmaceuticals and Petra Pharmaceuticals, companies developing drugs for treating cancer. The laboratory of L.C. Cantley also receives funding from Petra. A.B. Hanker receives grant support from Takeda. A.L Guerrero-Zotano has received grant support from Pfizer and travel support from Pfizer and Roche. V.M. Jansen is a current employee and shareholder of Eli Lilly and Company.

\section{Additional information}

Supplementary information is available for this paper at https://doi.org/10.1038/s41467020-19291-x.

Correspondence and requests for materials should be addressed to C.L.A.

Peer review information Nature Communications thanks the anonymous reviewer(s) for their contribution to the peer review of this work. Peer reviewer reports are available.

Reprints and permission information is available at http://www.nature.com/reprints

Publisher's note Springer Nature remains neutral with regard to jurisdictional claims in published maps and institutional affiliations.

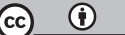

Open Access This article is licensed under a Creative Commons Attribution 4.0 International License, which permits use, sharing, adaptation, distribution and reproduction in any medium or format, as long as you give appropriate credit to the original author(s) and the source, provide a link to the Creative Commons license, and indicate if changes were made. The images or other third party material in this article are included in the article's Creative Commons license, unless indicated otherwise in a credit line to the material. If material is not included in the article's Creative Commons license and your intended use is not permitted by statutory regulation or exceeds the permitted use, you will need to obtain permission directly from the copyright holder. To view a copy of this license, visit http://creativecommons.org/ licenses/by/4.0/.

(C) The Author(s) 2020 